\title{
Thermal Study of a Cladding Layer of Inconel 625 in Directed Energy Deposition (DED) Process Using a Phase-Field Model
}

Roya Darabi ( $\sim$ rdarabi@inegi.up.pt )

Institute of Science and Innovation in Mechanical and Industrial Engineering (INEGI)

André Ferreira

Institute of Science and Innovation in Mechanical and Industrial Engineering (INEGI)

Erfan Azinpour

Faculty of Engineering of University of Porto (FEUP)

Jose Cesar de Sa

Institute of Science and Innovation in Mechanical and Industrial Engineering (INEGI)

Ana Reis

Institute of Science and Innovation in Mechanical and Industrial Engineering (INEGI)

\section{Research Article}

Keywords: DED, Additive manufacturing, FEM, Phase-field method, Melt pool morphology, Solidification

Posted Date: September 13th, 2021

DOI: https://doi.org/10.21203/rs.3.rs-866393/v1

License: (9) This work is licensed under a Creative Commons Attribution 4.0 International License. Read Full License

Version of Record: A version of this preprint was published at The International Journal of Advanced Manufacturing Technology on January 11th, 2022. See the published version at https://doi.org/10.1007/s00170-021-08376-6. 


\title{
Thermal study of a cladding layer of Inconel 625 in Directed Energy Deposition (DED) process using a phase-field model
}

\author{
Roya Darabi*1,2 ${ }^{*}$ André Ferreira ${ }^{1,2}$, Erfan Azinpour ${ }^{2}$, Jose Cesar de Sa ${ }^{1}$, Ana Reis ${ }^{1,2}$ \\ ${ }^{1}$ Institute of Science and Innovation in Mechanical and Industrial Engineering (INEGI), FEUP campus, Rua Dr. \\ Roberto Frias, 400, 4200-465, Porto, Portugal \\ ${ }^{2}$ Faculty of Engineering of University of Porto (FEUP), Rua Dr. Roberto Frias, 4200-465, Porto, Portugal \\ *Corresponding Author: rdarabi@inegi.up.pt
}

\begin{abstract}
In an effort to simulate the involved thermal physical effects that occur in direct energy deposition (DED) a thermodynamically-consistent of phase-field method is developed. Two state parameters, characterizing phase change and consolidation, are used to allocate the proper material properties to each phase. The numerical transient solution is obtained via a finite element analysis. A set of experiments for single tracks scanning were carried out to provide dimensional data of the deposited cladding lines. By relying on a regression analytical formulation to establish the link between process parameters and geometries of deposited layers from experiments, an activation of passive elements in the finite element discretization is considered. The single-track cladding of Inconel 625 powder on tempered steel $42 \mathrm{CrMo} 4$ was printed with different power, scanning speed and feed-rate to assess their effect on the morphology of the melt pool and the solidification cooling rate. The predicted dimensions of melt pools were compared with experiments reported in the literature. In addition, this research correlated the used process parameter in the modelling of localized transient thermal with solidification parameters, namely, the thermal gradient $(G)$ and the solidification rate $(R)$.
\end{abstract}

Keywords: DED. Additive manufacturing. FEM. Phase-field method. Melt pool morphology. Solidification 


\section{Introduction}

Additive manufacturing (AM) is a transformative approach to industrial production that enables the creation of lighter and stronger parts with higher flexibility in the design to achieve desirable mechanical properties and high dimensional accuracy. The AM processes consolidate feedstock materials such as powder, wire or sheets into a dense metallic part by melting and solidification with the aid of an energy source such as laser, electron beam or electric arc, in a layer by layer manner[1][2]. Directed Energy Deposition (DED) covers a range of terminology including laser engineered net shaping products, directed light fabrication, direct metal deposition and 3D laser cladding [3]. DED is a complex printing process commonly used to repair or add additional material to existing components [4][5]. DED involves injecting a stream of metallic powder that is melted by a laser beam as a heat source in order to deposit material layer-by-layer on a built platform [6]. In DED process, solidification and solid-state transformations, upon heating and cooling, deeply affect the mechanical properties of deposited layer induced by the high-energy input and high cooling rate during the process [7]. The control of the involved physical phenomena like melting, phase changing, vaporization, and Marangoni convection is extremely difficult and sometimes impossible exclusively by means of experimental analyses [8]. Furthermore, it is rather time-consuming and expensive to produce DED fabricated parts. Computational simulation can give precious information on the complex process-structure-property relations and therefore be useful to its design and optimization. The phenomenon has a multi-scale inherent nature which is computationally complex and challenging that, so far, must be tackled at different stages. Temperature prediction and solid-liquid phase fields detection can be defined as the initial stage in the process simulation, that can then be utilized subsequently in thermal-mechanical and material microstructure evolution. The model needs to properly consider the material properties with respect to solid-liquid phase changing, powder-dense material status. Lee et al. presented enhanced models for temperature evolution and phasedependent thermal conductivity and heat capacity in selective laser melting (SLM) process [9][10]. Roy et al. proposed a purely thermal model which explicitly incorporates two state variables for both the phase and the porosity in SLM. Their model has the ability to capture the consolidation of the material and allowed them to investigate the phase-dependent laser absorptivity [11]. It is worth noting that although a broad number of numerical modeling approaches for selective laser melting (SLM) can be found in recent years, capable of predicting the temperature field, melting and solidification [12] [13], there is a lack of the detailed studies on phase detecting on DED. The origin of this inattention comes from another existing challenge in DED simulation, including the dynamic incorporation of the additive material as the deposited layer into the numerical model. Through activation of a new set of elements in each time step of the finite element solution. Moreover, DED process has a highly localized thermal 
behavior which leads to undesirable microstructural features [14] and inconsistent mechanical properties of the fabricated parts. Nevertheless, some significant efforts have been made to simulate solidification kinetics [15] and investigation results on the correlation microstructural features and mechanical properties between thermal characterization of melt pool and solidification parameters, including thermal gradient $G$ and solidification rate, have been reported [16]. The effect of increasing the laser speed and decreasing the power simultaneously on the melt pool size, thermal gradients and cooling rates were illustrated in [17]. Correlation between solidification parameters which can be derived from numerical thermal models based on the Finite Element Method (FEM) were reported in [18][19]. Subsequently, the microstructure was predicted using the solidification map of the specific material. Finally, indirect microstructure control was achieved by relating the predicted microstructure to the derived melt pool dimensional map. To the best of the authors' knowledge, very little attention has been given, so far, to the development of a transient heat numerical model on DED process involving phase changing to predict of the melt pool boundary with varying laser power to extract the solidification parameters.

The present article focuses on the development of a thermal powder deposition evolution for DED process using the commercial FEM software ABAQUS. The transient heat transfer model associated with the phase field concept is implemented by user coding in FORTRAN language, taking into consideration the latent heat of fusion and vaporization. In the present model, the volume fraction of the deposited material is modeled based on the synergistic interactions from experiment-driven equations. Subsequently, the validation procedure is carried out based on experimentally measured melt pool dimensions related to single track fabrication of IN 625 on a $42 \mathrm{CrMo} 4$ baseplate. Then the calculated solidification parameters $(G, R)$ were compared across the melt pool by changing laser power to shed light on its effect on the microstructure map.

The paper is organized as follows. Section 2 contains the numerical approach consisting of governing equations, describing the transient heat transfer model associated with the phase change concept, material allocations for both deposition and substrate with the concept of the phase-field model and heat source modeling. Section 3 presents the experimental study for single-track lines of IN625 on the tempered substrate $42 \mathrm{CrMo} 4$ with different power, scanning speed and feed rate to achieve various penetrations. In section 4, the thermal phenomena based on finite element formulation is implemented in ABAQUS through relevant user interface routines. Subsequently, in section 5 the results from the proposed numerical method are compared with experimental data to assess the efficiency of the model. In Section 6, conclusions are drawn and summarized. 


\section{Proposed Numerical Approaches}

The direct energy deposition (DED) process modelling is presented in detail in the following sections, in terms of heat transfer constitutive equations, allocated material properties with respect to temperature and material states and energy source modelling;

\subsection{Governing equation of thermal energy balance}

The thermal energy balance equation based on first law of thermodynamic states that change in the total energy equals to the rate of work, which is done on the volumetric boundary of $\Omega$. The adaption of energy balance considering the phase parameters, temperature, flux boundary and initial conditions are represented in Equations.1-3 [20][9][11];

\begin{tabular}{|ll|c|}
\hline$\frac{d e}{d t}=\nabla \cdot[k(\psi) \nabla(T)]+U(\boldsymbol{x}, t)$ & in $\Omega$ & (1) \\
\hline$-[k(\psi) \nabla(T)] \cdot \boldsymbol{n}=\hat{q}$ & on $\Gamma_{2}$ & (2) \\
\hline$\hat{q}=h\left(T-T_{0}\right)+\varepsilon \sigma\left(T^{4}-T_{0}^{4}\right)$ & (3) \\
\hline
\end{tabular}

where $e, k, U$ are energy density, thermal conductivity and the volumetric heat source delivered from laser beam. with $\hat{q}$ prescribed heat flux vector on the surface boundary, which can include the heat loss for convection and radiation terms and $\boldsymbol{n}$ is the outward unit normal vector. The parameters $\varepsilon=$ $0.28, h=10\left(\frac{W}{m^{2} K}\right)$ and $\sigma=5.67 \times 10^{-8}\left(\frac{W}{m^{2} K^{4}}\right)$ are the emissivity of the material, heat convection and coefficient Stefan-Boltzmann constant respectively. In addition, the value of, 10 and $5.67 \times 10^{-8}$ are dedicated to these values.

The energy density is expressed in terms of the temperature and state variables as below;

$e=C_{s}(\psi) T+p\left(\phi_{f}\right)\left\{L_{f}+\left[C_{l}-C_{s}(\psi)\right]\left(T-T_{m}\right)\right\}+L_{v} p\left(\phi_{v}\right) \quad$ in $\Omega$

Here $C_{S}, C_{l}$ and $L_{f}, L_{v}$ are the volumetric heat capacities in the solid and liquid states, the latent heat of fusion-melting [21][22] and latent heat of vaporization respectively. $T_{m}$ is the average melting temperature, taken as $T_{m}=0.5 *\left(T_{s}+T_{l}\right)$, where $T_{l}$ and $T_{s}$ are liquidus and solidus temperatures [23].

The used function $p\left(\phi_{\text {for } v}\right)$ in Equation 4 is defined based on the thermodynamically consistent phase-field approach proposed by Wang et al. [20] such that $p(0)=0$ and $p(1)=1$ and $\frac{d p}{d \phi_{\text {for } v}}=$ 
$\frac{d^{2} p}{d^{2} \phi_{\text {for } v}}=0$ at $\phi_{\text {for } v}=0$ and $\phi_{\text {for } v}=1$ [23], where $\phi_{\text {for } v}$ is the phase parameter. It takes the following form:

$p\left(\phi_{\text {f or } v}\right)=\left(\phi_{\text {f or } v}\right)^{3}\left[10-15\left(\phi_{\text {f or } v}\right)+6\left(\phi_{\text {f or } v}\right)^{2}\right]$

In Equation 5 the phase parameters $\phi_{f}, \phi_{v}$ are defined as:

$$
\begin{aligned}
& \phi_{f}=\frac{1}{2}\left(\tanh \left(A\left(\frac{T-T_{m}}{T_{l}-T_{S}}\right)+1\right)\right) \\
& \phi_{v}=\frac{1}{2}\left(\tanh \left(A\left(\frac{T-T_{v}}{T_{v p}-T_{v l}}\right)+1\right)\right)
\end{aligned}
$$

where $T_{v p}$ is the vaporized temperature, $T_{v l}$ is the temperature at the liquid-vapor transition and $T_{v}$ is the average vaporization temperature taken as $T_{v}=0.5 *\left(T_{v l}+T_{v p}\right)$.

Some conditions are considered: $\phi_{f}=0$ if $T<T_{s}$ and $\phi_{f}=1$ if $T_{l}<T$. When $T_{S} \leq T \leq T_{l}$ then $0<\phi_{f}<1$, representing the mushy region. Moreover, $\phi_{v}=1$ if $T_{v p}<T$, indicating whether the material is vaporized or not. The parameter $A$ determines the steepness of change transition [10]. The effect of the choice of $A$ on the profile of the phase parameter $p\left(\phi_{f}\right)$ (for sharp transition $A=10$ and for diffuse transition $A=5$ ) is shown in Figure. 1.

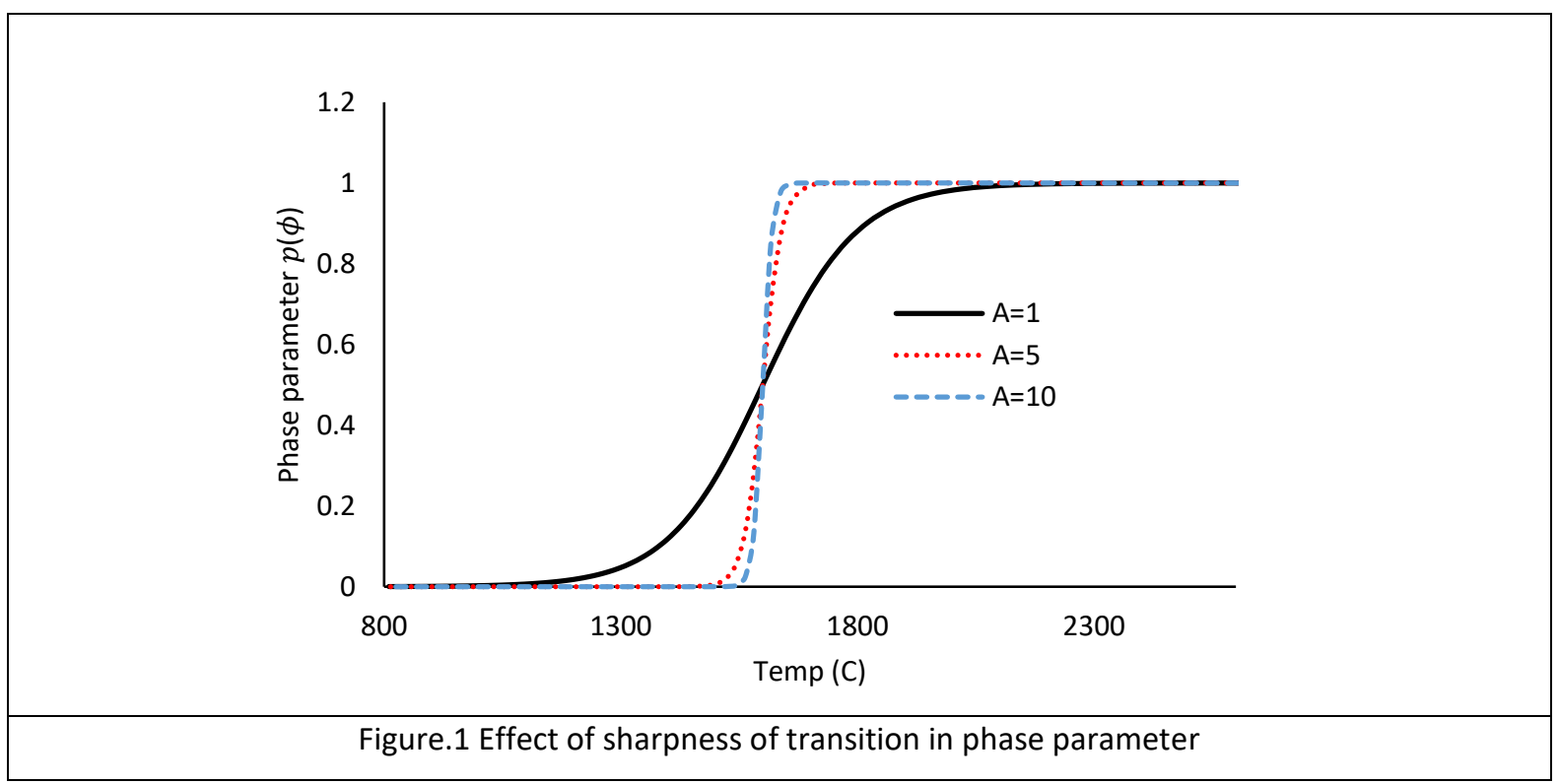

Regarding Equation 1, the thermal properties of the material, including volumetric heat capacity and thermal conductivity, are defined based on the consolidation parameter $\psi$ as in Equation 8. This 
parameter always keeps the maximum values of $\phi_{f}$ to characterize the thermal history at each material point.

$\psi(\boldsymbol{x}, t)=\max \left\{\phi_{f}(\boldsymbol{x}, t+d t), \psi(\boldsymbol{x}, t)\right\}$

When $\psi=0$ the material is still in the powder state and $\psi=1$ refers to the fully dense region. Table 1 shows the material state relation with the state variables.

Table 1. $\phi_{f}(T), \psi(T)$ values for different states of the material

\begin{tabular}{|c|c|c|c|c|c|}
\hline Powder & Solid porous & Mushy powder-melt & Liquid melt & Mushy melt-dense & Solid dense \\
\hline$\phi_{f}(T)=0$ & $\phi_{f}(T)=0$ & $0<\phi_{f}(T) \leq 1$ & $\phi_{f}(T)=1$ & $0 \leq \phi_{f}(T) \leq 1$ & $\phi_{f}(T)=0$ \\
\hline$\psi(T)=0$ & $0<\psi(T)<1$ & $0<\psi(T) \leq 1$ & $\psi(T)=1$ & $\psi(T)=1$ & $\psi(T)=1$ \\
\hline
\end{tabular}

According to Equation 8, $\psi$ which is related to the history of the fusion phase parameter at each material point. The mentioned volumetric heat capacity $C_{s}(\psi)$ and thermal conductivity $k(\psi)$ in Equations 1 and 2 are determined by the degree of consolidation $(\psi)$ defined in Equations 9-10

\begin{tabular}{|l|c|}
\hline$k(\psi)=(1-\psi) k_{p}+\psi k_{d}$ & (9) \\
\hline$C_{s}(\psi)=\left(1-\varepsilon_{0}(1-\psi)\right) C_{d}$ & (10) \\
\hline
\end{tabular}

where $k_{p}$ and $k_{d}$ are the thermal conductivity in the powder and dense material, respectively. The volumetric heat capacity depends on the consolidation $\psi$ and $C_{d}$, the latter being the heat capacity of the fully dense material and $\varepsilon_{0}$ represents the initial porosity of powder and assumed 0.6. In general, the thermal conductivity and heat capacity are also temperature dependent. In the next section, the correlation between materials' thermo-physical properties with state variables are presented.

\subsection{Material Properties Module}

Nickel-based super alloy powder (MetcoClad625 ${ }^{\circledR}$ ) as cladding powder and $42 \mathrm{CrMo} 4$ tempering steel as substrate are considered in this research. MetcoClad625 is used as a blown powder cladding layer and tempering steel is employed as a solid substrate part. Figures 2 and 3 along with the Table 2 represent the thermo-physical material properties depending on the temperature for MetcoClad625 (both powder and solid phase) and steel $42 \mathrm{CrMo} 4$. In the DED process, a great part of the blown powder particle undergoes a phase change and turns into a liquid state by a heat source, while the rest of the material remains in mushy powder-melt state. Thereafter, as the material cools down, the melted parts change to a solid state. Since the difference of material properties between the liquid 
area with mushy zones and the solid state is very large, the current state of the material should be identified in order to utilize appropriate properties according to the phase change history.
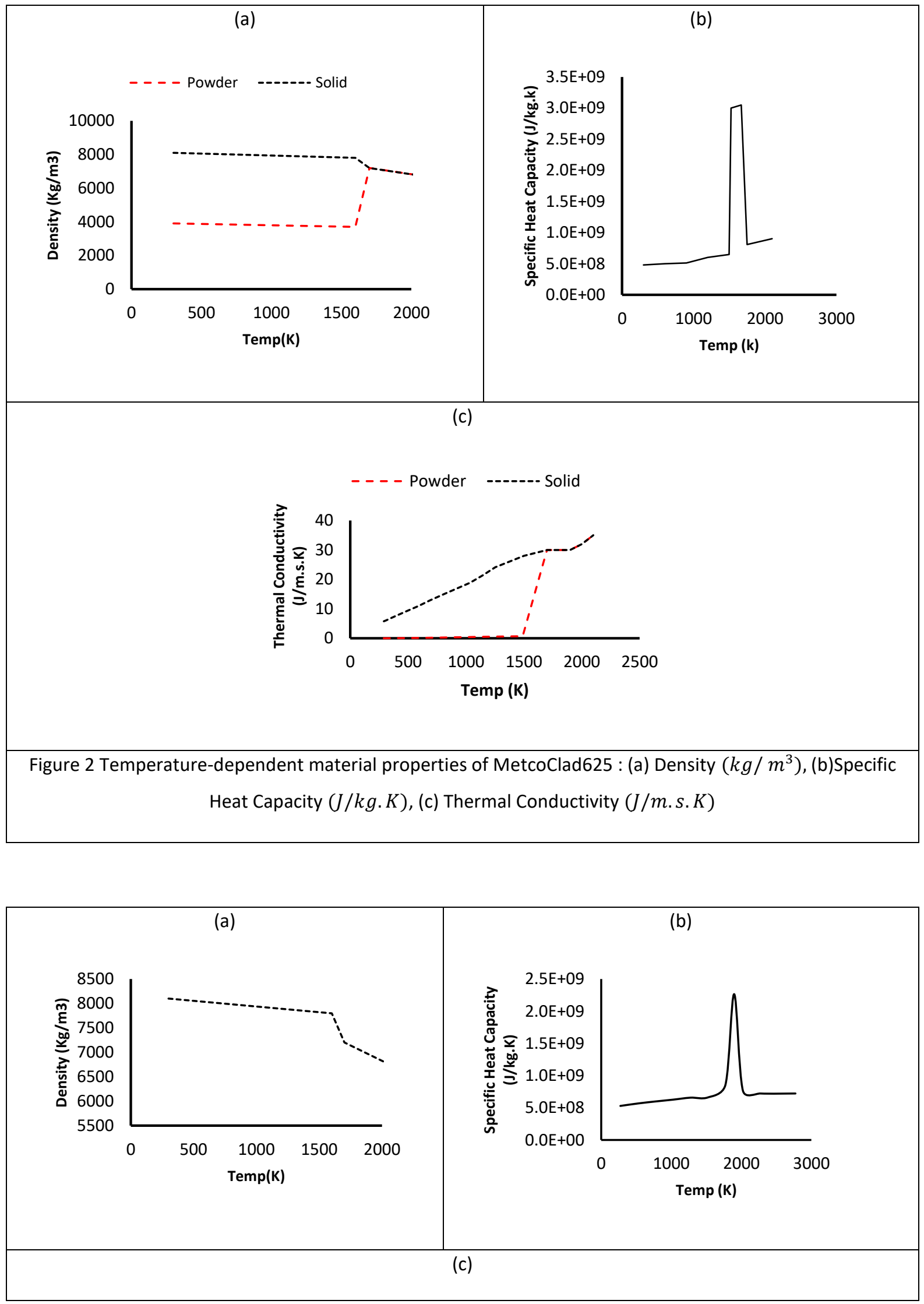


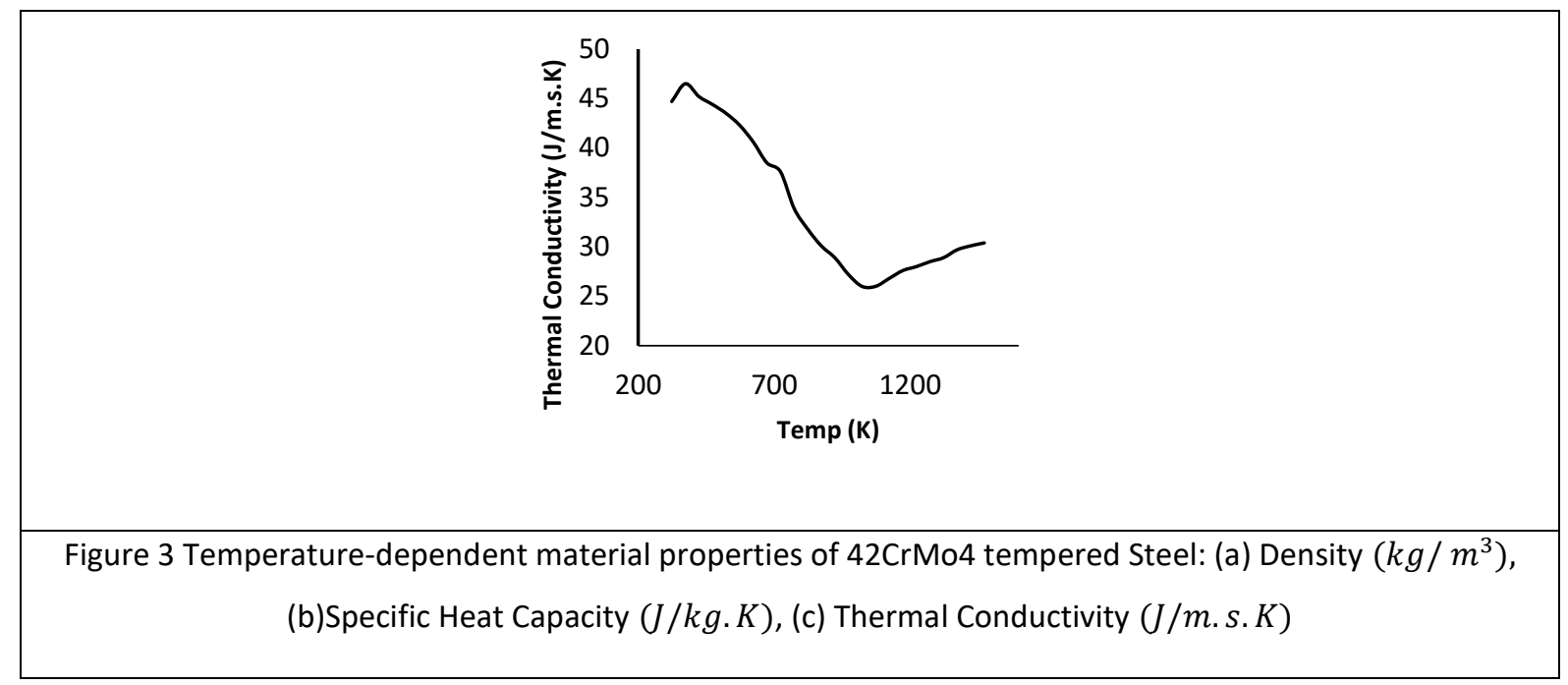

Table 2. Thermo-physical properties of the material

\begin{tabular}{|ccccccc|}
\hline & $T_{s}(K)$ & $T_{l}(K)$ & $L_{f}(\mathrm{~kJ} / \mathrm{kg})$ & $T_{v p}(K)$ & $T_{v l}(K)$ & $L_{v}(\mathrm{~kJ} / \mathrm{kg})$ \\
\hline MetcoClad625 & 1563 & 1723 & 44.34 & 3580 & 3650 & 91.3 \\
\hline 42 CrMo4 & 1770 & 1920 & 25.72 & 3560 & 3680 & 104 \\
\hline
\end{tabular}

Figure 4 shows the different phases throughout deposition process between solid and liquid phases.

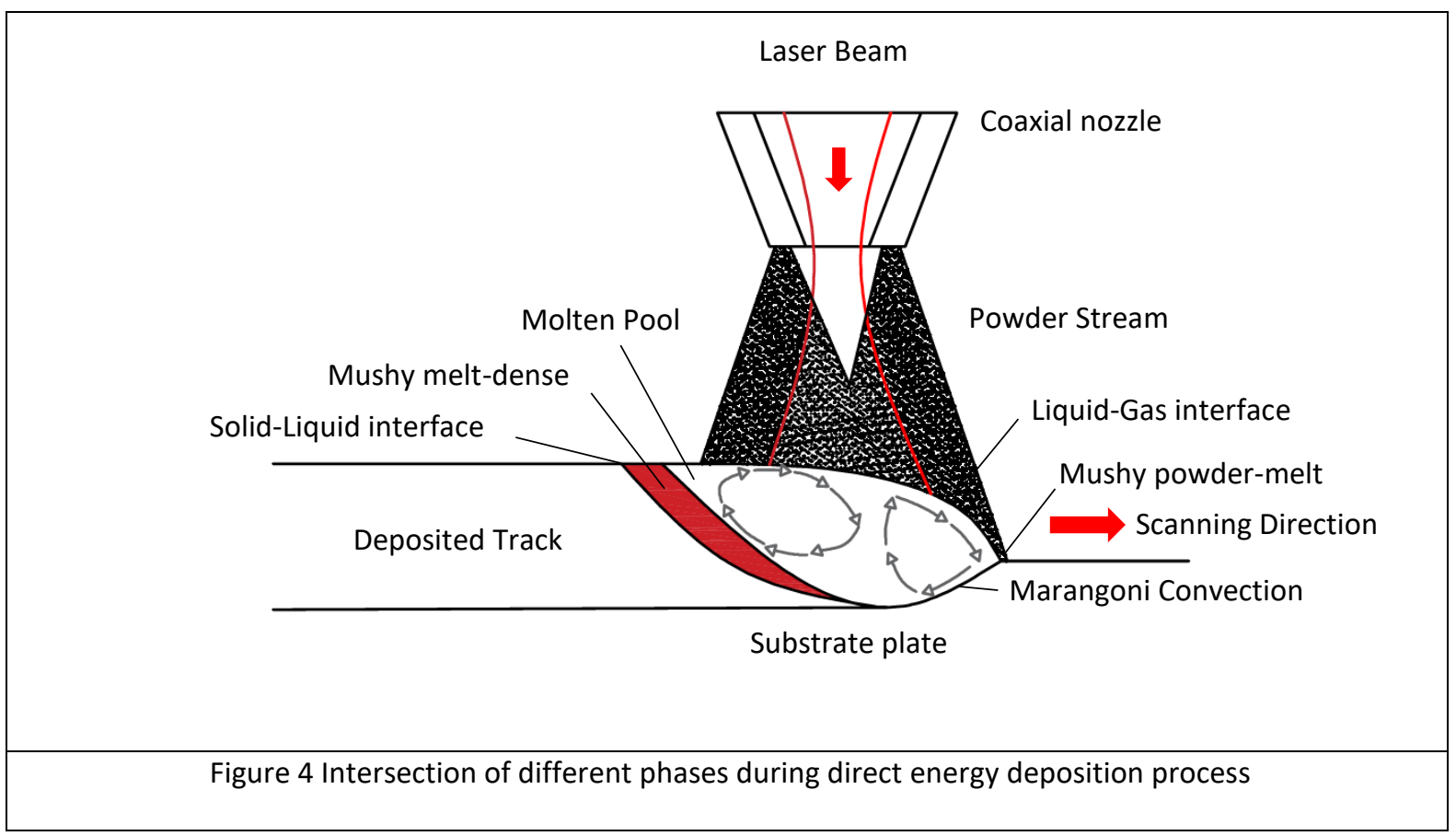

\subsection{Heat Source Model}

It is important to establish an appropriate heat source model of laser deposition simulations since the heat source not only influences the geometries of melt pools, but also it may have an impact on the 
mechanical performance of final products. Heat source models used in DED simulations are typically assumed [24][25], as the following two-dimensional exponentially decaying function of Equation 11 which is schematically represented in Figure 5,

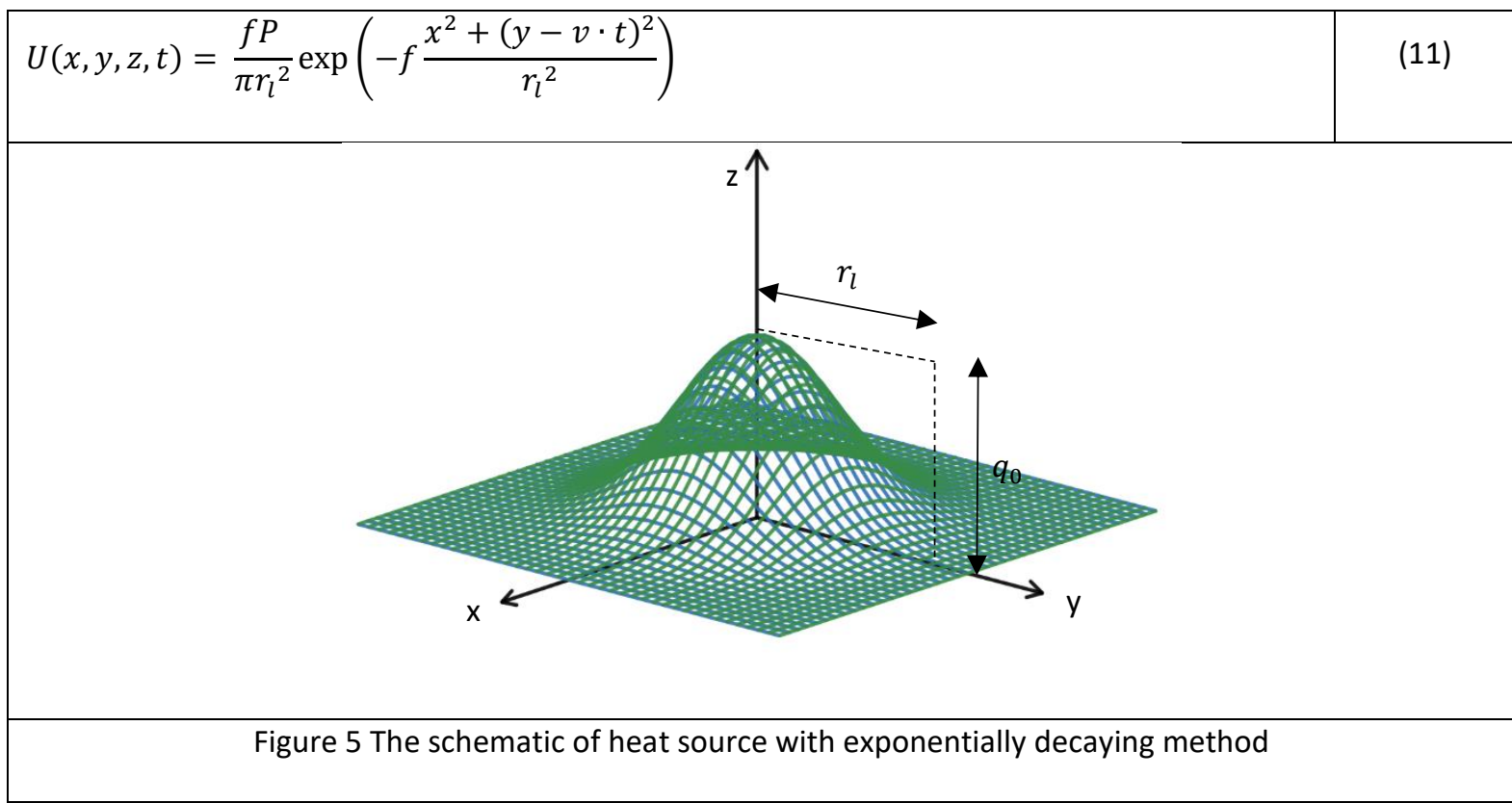

where $P$ is the laser power, $f$ is the distribution of power factor, $r_{l}$ is laser beam radius corresponding to the distance between the beam center and the point at coordinates $(x, y), v$ is the laser speed moving. Figure 6 illustrates the schematic profile of power density with respect to the distribution factor.

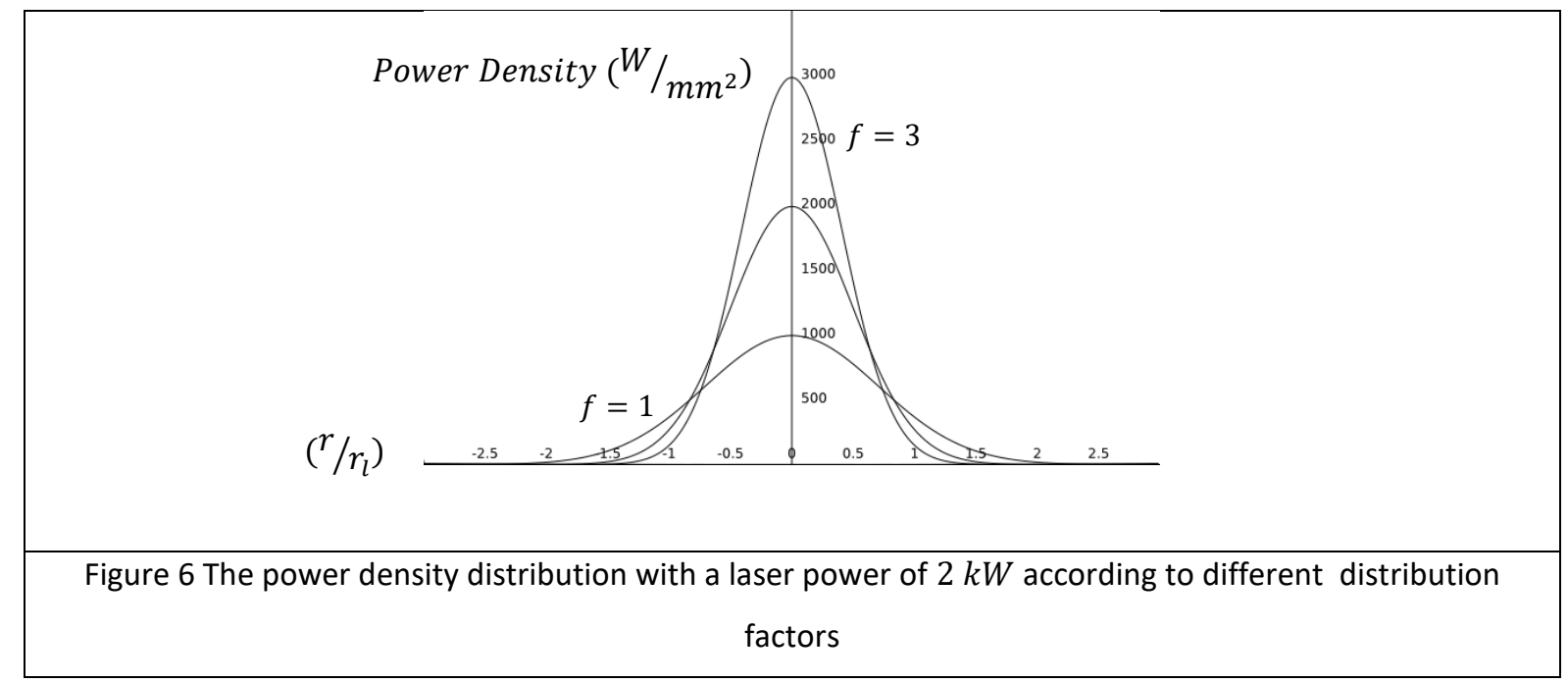




\section{Experimental procedure}

A tempered steel $(42 \mathrm{CrMo} 4)$ plate with size of $100 \mathrm{~mm} \times 120 \mathrm{~mm} \times 15 \mathrm{~mm}$ was used as a substrate. In the preparation process, the surface was machined and then cleaned by ethanol. The used powder is commercially the gas-atomised Nickel-based super alloy (MetcoClad 625), similar to Inconel 625. Figure 7 shows the micrography of the powder. Laser cladding experiments were performed by a coaxial laser machine "LDF 3000 - 100" with a fibre-coupled high power laser diode (adjustable wavelength 900-1030 $\mathrm{nm}$ that changes depend on power), with $6000 \mathrm{~W}$ maximum beam power output. The laser machine was equipped with a 6-axes KUKA KR90 R3100 industrial robot. Based on Table 3, 18 different single tracks, $15 \mathrm{~mm}$ in length, were produced by altering three process parameters, namely laser powers $\mathrm{P}(1,1.5,2,2.5,3 \mathrm{~kW})$, scanning speeds $v(2,4,6,10 \mathrm{~mm} / \mathrm{s})$ and powder feed rates $F(10,15,20 \mathrm{gr} / \mathrm{min})$. All other parameters remained constant throughout the experiments: the laser spot diameter was $2.5 \mathrm{~mm}$ with a top-hat beam profile. A high purity argon (99.99\%) as the shield gas, with a flow rate equal to $5.5 \mathrm{~L} / \mathrm{min}$, was utilized to minimize contamination and oxidisation. The single tracks were cross-sectioned, mounted in resin and polished down to $1 \mu \mathrm{m}$ diamond suspension as a last stage. The geometry measurements of height, depth and width of the cladding lines produced by the DED technique were achieved using a Leica DVM6 A 2019 digital microscope. Figure $8(\mathrm{a}-\mathrm{f})$ shows the geometrical measures of the clad section of the molten pool for some samples using software ImageJ. Table 3 presents the measured value from designed experiments for Inconel 625 single tracks cladded on 42CrMo4 entirely.

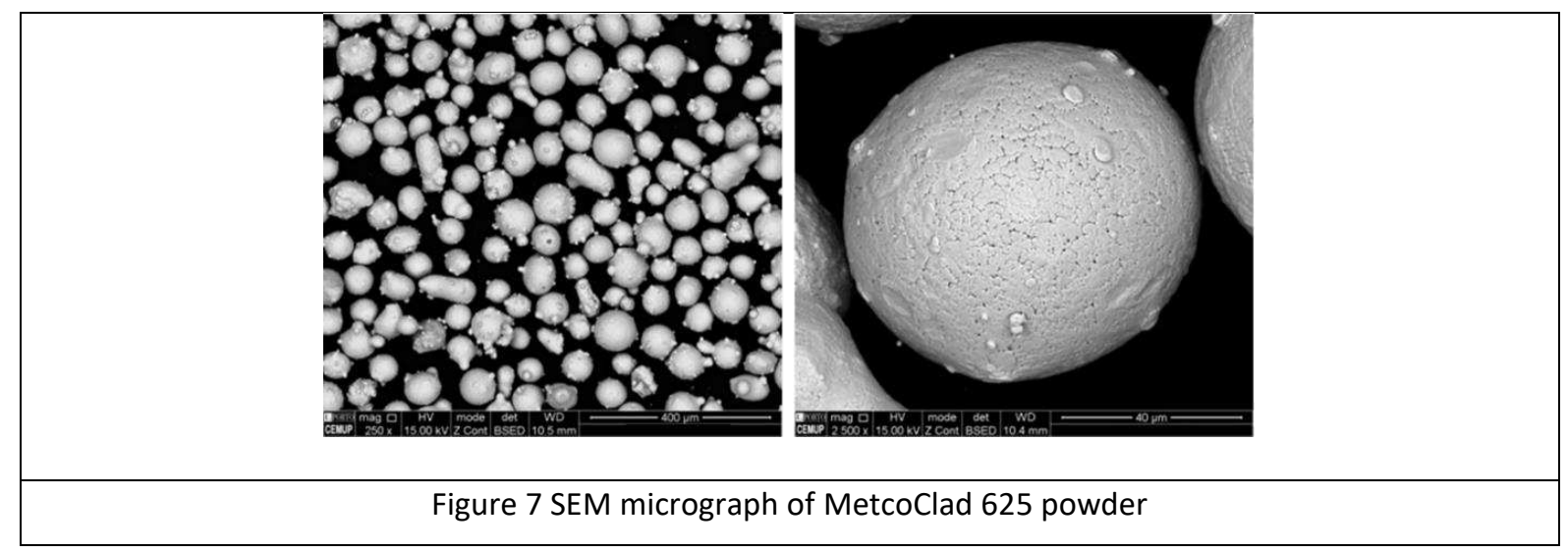




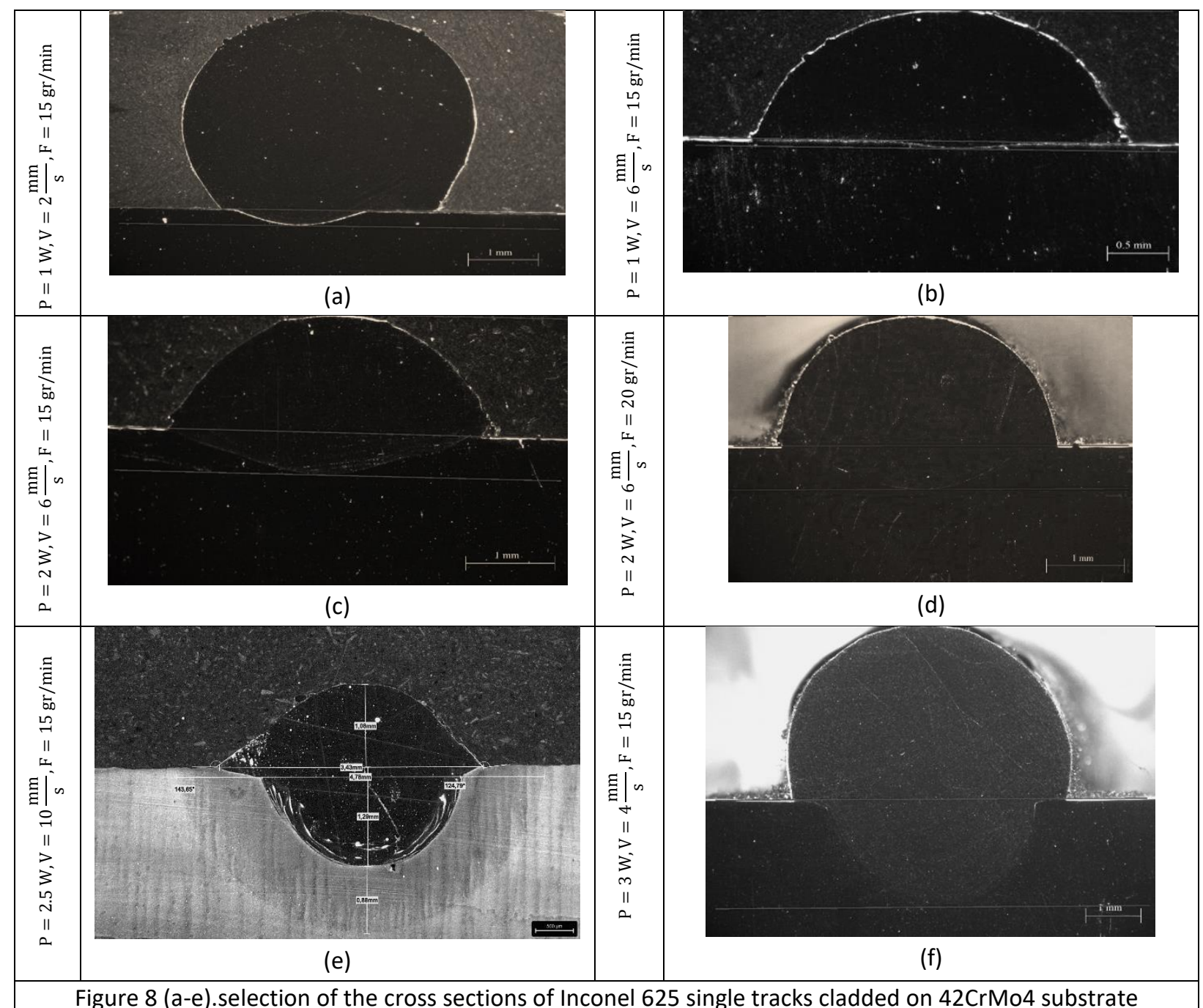

Table 3 Measured value sets for width, height and penetration depth in single clads Inconel 625 on $42 \mathrm{CrMo} 4$ substrate

\begin{tabular}{|c|c|c|c|c|c|c|c|c|c|}
\hline \#Set & 1 & 2 & 3 & 4 & 5 & 6 & 7 & 8 & 9 \\
\hline $\begin{array}{l}\text { Power-Velocity-Reed rate } \\
\qquad \text { (P-V-F) }\end{array}$ & 1_2_15 & 1_6_15 & 1.5_10_10 & 1.5_10_15 & 2_2_15 & 2_4_15 & 2_6_10 & 2_6_15 & 2_6_20 \\
\hline $\mathrm{w}(\mathrm{mm})$ & 2.79 & 3.07 & 3.09 & 3.11 & 3.62 & 3.5 & 3.38 & 3.61 & 3.63 \\
\hline$h(\mathrm{~mm})$ & 3.07 & 1.22 & 0.71 & 1.03 & 3.66 & 2.47 & 1.39 & 1.47 & 1.71 \\
\hline $\mathrm{d}(\mathrm{mm})$ & 0.25 & 0.06 & 0.43 & 0.23 & 1.28 & 1.12 & 1.16 & 0.51 & 0.56 \\
\hline \#Set & 10 & 11 & 12 & 13 & 14 & 15 & 16 & 17 & 18 \\
\hline $\begin{array}{l}\text { Power-Velocity-Reed rate } \\
\qquad \text { (P-V-F) }\end{array}$ & 2_10_10 & 2_10_15 & 2.5_10_10 & 2.5_10_15 & 3_2_15 & 3_4_15 & 3_6_10 & 3_6_15 & 3_6_20 \\
\hline$w(\mathrm{~mm})$ & 3.2 & 3.36 & 3.33 & 3.46 & 5.05 & 4.64 & 3.89 & 4.71 & 3.94 \\
\hline$h(\mathrm{~mm})$ & 1.2 & 0.81 & 0.86 & 1.23 & 3.4 & 2.06 & 1.85 & 0.78 & 1.55 \\
\hline$d(\mathrm{~mm})$ & 0.72 & 0.88 & 1.04 & 1 & 2.06 & 0.89 & 1.08 & 0.68 & 1.34 \\
\hline
\end{tabular}


The specific energy $E$ and powder density $D$ are calculated via Equations 12 and 13 , where $P, D_{l}, v$ and $F$ are laser power, laser diameter, scanning speed and scanning feed rate respectively.

\begin{tabular}{|l|c|}
\hline$E\left(k W / m^{2}\right)=P / D_{l} * v$ & (12) \\
\hline$D\left(g r / m m^{2}\right)=F / D_{l} * v$ & (13) \\
\hline
\end{tabular}

Then, the synergistic interaction between regression-based specific energy and powder density can be represented by Equations 14 and 15 . Adjusted R-square values are $86.55 \%$ and $75.07 \%$ respectively for $H$ (height of cladding layer) and $W$ (width of cladding layer).

\begin{tabular}{|l|l|}
\hline$H(m m)=0.51761+0.00025 E+0.01083 D$ & (14) \\
\hline$W(m m)=3.12365+0.00127 E-0.00654 D$ & (15) \\
\hline
\end{tabular}

\section{Numerical implementation with finite element method (FEM)}

To obtain the thermal model for DED process, including subsequent results such as temperature, melt pool dimensions, interfacial phenomena, a thermal finite element analysis framework was built using the commercial software ABAQUS/Standard. ABAQUS provides the interface for mesh designing, programming user-defined material behavior and boundary conditions. Hereby, the specific features and numerical models of DED introduced in Section 2 associated with experimental results, in section 3 , are implemented using provided user subroutines as follows.

\subsection{Finite element solution for heat transfer}

Using the Galerkin weighted residual method it is possible to obtain from Equations 1 and 2 the following classical integral (weak) form as Equation 16;

$$
\int_{\Omega}(w \dot{e}(T)+\nabla w \cdot k(T) \nabla T-w U) d \Omega+\int_{\Gamma_{2}} w \cdot \hat{q} d \Gamma_{2}=0
$$

where $w=w(\boldsymbol{x})$ is a weighting function, and from which, utilizing the finite element method, a system of ordinary differential equations can be written, in a matrix form as Equation 17:

$C(T) \dot{T}+K(T) T=F$

In Equation 17, $\boldsymbol{T}$ is the nodal temperature vector, $\dot{\boldsymbol{T}}$ its time derivative, $\boldsymbol{C}(\boldsymbol{T})$ a temperature dependent equivalent capacity matrix, resulting terms that include temperature time derivative in 
Equation $16, \boldsymbol{K}(\boldsymbol{T})$ is the equivalent conductivity matrix, resulting from terms that include temperature in Equation 16 and $\boldsymbol{F}$ is the equivalent time dependent heat source, resulting from independent terms in Equation 16.

Using an implicit time integration scheme to solve Equation 17, in which it is assumed that

$$
\dot{\boldsymbol{T}}^{t+\Delta t}=\frac{1}{\Delta t}\left(\boldsymbol{T}^{t+\Delta t}-\boldsymbol{T}^{t}\right)
$$

Then final nonlinear system of equations to be solved results in Equation 19;

$$
\boldsymbol{C} \boldsymbol{T}^{t+\Delta t}+\Delta t \boldsymbol{K} \boldsymbol{T}^{t+\Delta t}=\Delta t \boldsymbol{F}^{t+\Delta t}+\boldsymbol{C} \boldsymbol{T}^{t}
$$

The nonlinear system of equations is iteratively solved, within each time step, resorting to the Newton method, in a three-step procedure at each iteration $i$ as below;

\begin{tabular}{|l|c|}
\hline $\boldsymbol{R}_{i}=\boldsymbol{C} \boldsymbol{T}_{i}^{t+\Delta t}+\Delta t \boldsymbol{K} \boldsymbol{T}_{i}^{t+\Delta t}-\Delta t \boldsymbol{F}^{t+\Delta t}-\boldsymbol{C} \boldsymbol{T}^{t}$ & (20) \\
\hline$\left(\frac{\partial \boldsymbol{C}}{\partial \boldsymbol{T}}+\Delta t \frac{\partial \boldsymbol{K}}{\partial \boldsymbol{T}}\right) \Delta \boldsymbol{T}_{i}=-\boldsymbol{R}_{i}$ & (21) \\
\hline $\boldsymbol{T}_{i+1}^{t+\Delta t}=\boldsymbol{T}_{i}^{t+\Delta t}+\Delta \boldsymbol{T}_{i}$ & (22) \\
\hline
\end{tabular}

Information on the residual force vector of Equation 20 and on the Jacobian matrix in Equation 22 must be given in the implementation of the method in ABAQUS. In particular a special care must be taken on the information from the definition of the terms resulting from the variation of internal energy density with temperature, $\frac{d e}{d T}$, as depending on whether the value of $\psi$ parameter is updated or not, the following energy density equations should be utilized as Equations 23 and 24:

$$
\begin{aligned}
& \frac{d e}{d T}=C_{s}(\psi)+ \frac{d p\left(\phi_{f}\right)}{d T}\left\{L_{f}+\left[C_{l}-C_{s}(\psi)\right]\left(T-T_{m}\right)\right\}+p\left(\phi_{f}\right)\left(C_{l}-C_{s}(\psi)\right) \\
&+L_{v} \frac{d p\left(\phi_{v}\right)}{d T} \\
& \frac{\frac{d e}{d T}=\frac{d C_{s}\left(\phi_{f}\right)}{d T} T}{}+C_{s}\left(\phi_{f}\right)+\frac{d p\left(\phi_{f}\right)}{d T}\left\{L_{f}+\left[C_{l}-C_{s}\left(\phi_{f}\right)\right]\left(T-T_{m}\right)\right\} \\
&-p\left(\phi_{f}\right) \frac{d C_{s}\left(\phi_{f}\right)}{d T}\left(T-T_{m}\right)+p\left(\phi_{f}\right)\left(C_{l}-C_{s}\left(\phi_{f}\right)\right) L_{v} \frac{d p\left(\phi_{v}\right)}{d T}
\end{aligned}
$$




\subsection{Implementation}

At present, there is no ready constitutive model in ABAQUS suitable for additive manufacturing simulation. The material phase change and taking into account the latent heat whether for fusion or vaporization into the thermal analysis, adding the deposited layers and the moving laser heat source are applied by resorting the user-coded subroutines programmed in FORTRAN language. The user subroutine UEPACTIVATIONVOL is utilized to prescribe and update the following variables: height and width of cladding layer. Then, the user-defined subroutine DFLUX is called to define the non-uniform distributed heat source $(U)$. In line with purpose of apply thermal constitutive behavior UMATHT subroutine is used and following variables need to update incrementally;

- Internal thermal energy (enthalpy) per unit of mass: $e$

- Derivative of internal energy with respect to the temperature: $\frac{d e}{d T}$

- Heat flux vector with respect to temperature: $\hat{q}=-k(\psi) \nabla(T)$

- Variation of heat flux vector to temperature: $\frac{\partial \hat{q}}{\partial T}$

- Variation of heat flux vector to the spatial gradient of temperature: $\frac{\partial \hat{q}}{\partial \frac{\partial T}{\partial x}} \quad(i=1,2,3)$

Therefore, the material state can be defined by the state variables $\phi_{\text {for } v}, \psi$ with respect to temperature. Finally, a USDFLD subroutine is developed to manage the material states at the end of each time increment. In Figure 9, a general flowchart summarizes the structure combination of used subroutines in ABAQUS.

\subsection{Time and space discretization}

The dimensional size of the modeled substrate is $100 \mathrm{~mm} \times 120 \mathrm{~mm} \times 15 \mathrm{~mm}$ and the cladding lines are modeled with $5 \mathrm{~mm} \times 5 \mathrm{~mm} \times 100 \mathrm{~mm}$ dimensions above the substrate as shown schematically in Figure 9. During the simulation, only a specific volume of the cladding line is activated based on the fed process parameters. This methodology makes the affordable balance for the computational time with the resolution of the results, thus making the simulation times reasonable. In this research, for both deposition layer and substrate, 3D thermal finite element mesh DC3D8 is utilized. The resolution of FEM model was selected to be high enough guarantee stabilization as well as an accurate cooling rate but keeping an affordable computational time. Thus artificial dispersion control introduces a stability limit on the size of the time increment and mesh size such that the local Courant number as Equation $25[26]$.

$C=|v| \frac{\Delta t}{\Delta x}$ meanwhile $C \leq 1$ 
where $|v|$ is the velocity and $\Delta t$ and $\Delta x$ represent the time increment and characteristic element size in the direction of flow respectively. The larger elements were used far away from the scanned region to reduce calculation time in the substrate. Meanwhile, to ensure a good link between the clad layers surface and the substrate, the mesh is refined as much as to avoid numerical temperature fluctuation due to very high temperature gradient.

\subsection{Initial and Thermal boundary condition}

All the surfaces of the cladding welding line and the substrate were initially fixed at $T_{0}=298.15 \mathrm{~K}$ and the sink temperature was also fixed at $T_{0}$ in the bottom of the baseplate. Besides, the tie constraint is applied between the top surface of baseplate and bottom surface of cladding layer to transfer the temperature between the contacted nodes. 


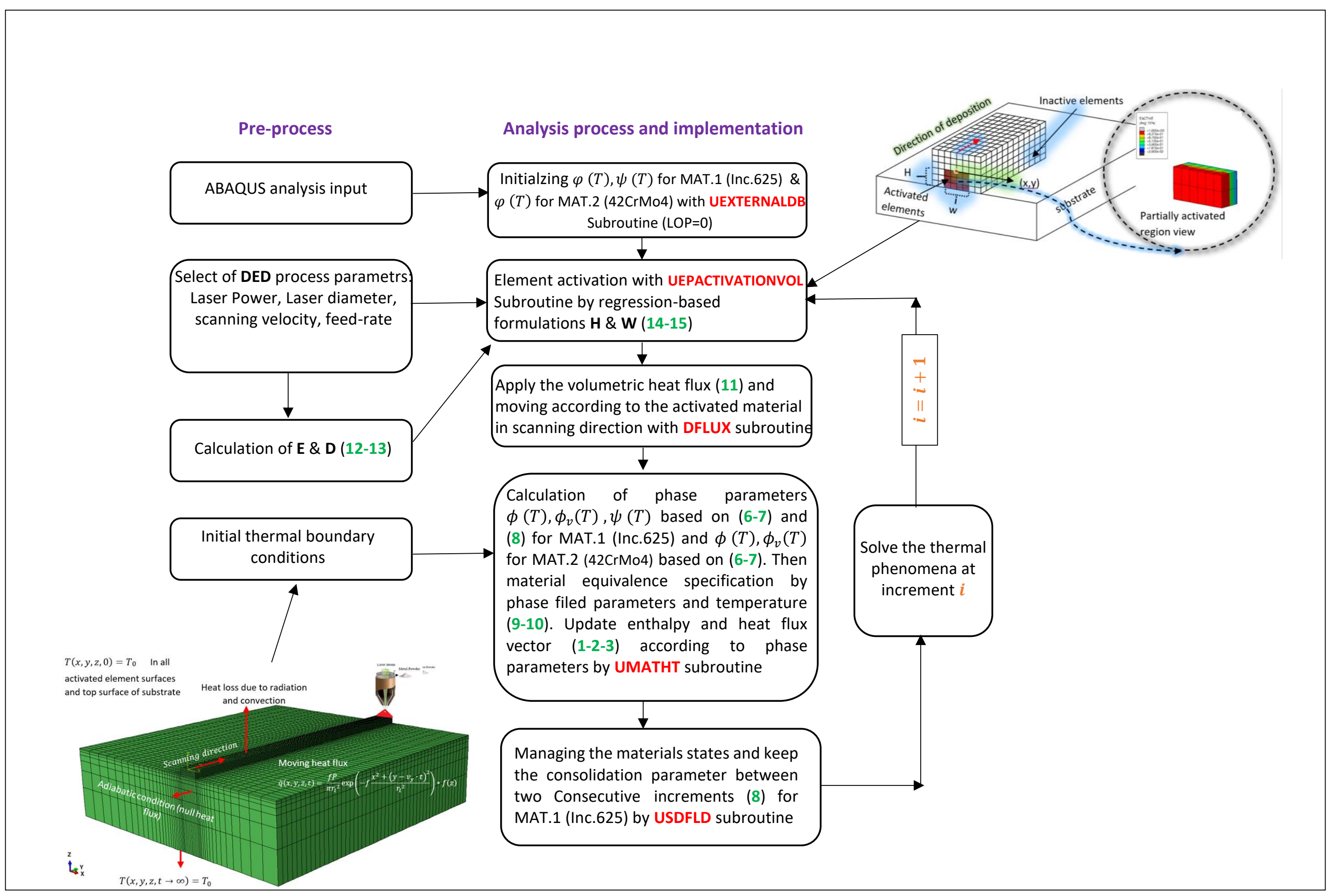

Figure $9 \mathrm{FE}$ model structure and the subroutine for the material addition (UEPACATIVATION VOL), the material state variable for the degree of solidification (USDFLD), and heat flux application (DFLUX), solving thermal material constitutive behavior (UMATHT) combinations 


\section{Result and discussion}

In this research, a numerical technique for the thermal part of DED process simulation using ABAQUS was proposed based on the phase-field concept. To demonstrate the thermal model and finite element implementation, firstly the data from experiments was used to predict of height and width of clad geometries, then compared the simulation in the area of melt pool with the experiments of the single laser tracks presented in section 3 . The estimation of the temperature field, the spatial variation of the melt pool morphology, the effect of the process parameters on the phase changing, and interfacial solidification were investigated. The analysis of the calculation results are summarized in the following sections:

\subsection{Sensitivity analysis to the time and space discretization}

The direct energy deposition (DED) processes commonly produce materials with heterogeneities on different length scales, which calls for a requirement of adopting adequate meshes and timescales in simulations in order to achieve accurate results. Previous studies [27][28] showed the effect of the mesh size and time increment in additive manufacturing simulation resolution results. Discretization with finer meshes improves the accuracy but increases the cost of computation and therefore assigning the proper mesh size and time-step is an essential requirement. Hereby, mesh sensitivity analysis is conducted for a cladding line with $P=1.5 \mathrm{~kW}, S S=10 \frac{\mathrm{mm}}{\mathrm{s}}$ and $F R=15 \mathrm{gr} / \mathrm{min}$. Figure 10 (a-b) shows a comparative temperature-location for the points of interest in the building direction and the width of cladding lines at a distance of $1 \mathrm{~mm}$ from the edge of the starting point of printing $(y=$ $1 \mathrm{~mm}$ ). Figure 10 (c) shows the temperature distribution, with time, for two grid refinement sizes with two time-steps. It reveals that sensitivity is greatly influenced by spatial discretization. The element size and Courant number were restricted to $0.2 \mathrm{~mm}$ and 1 respectively for the success of the simulation.

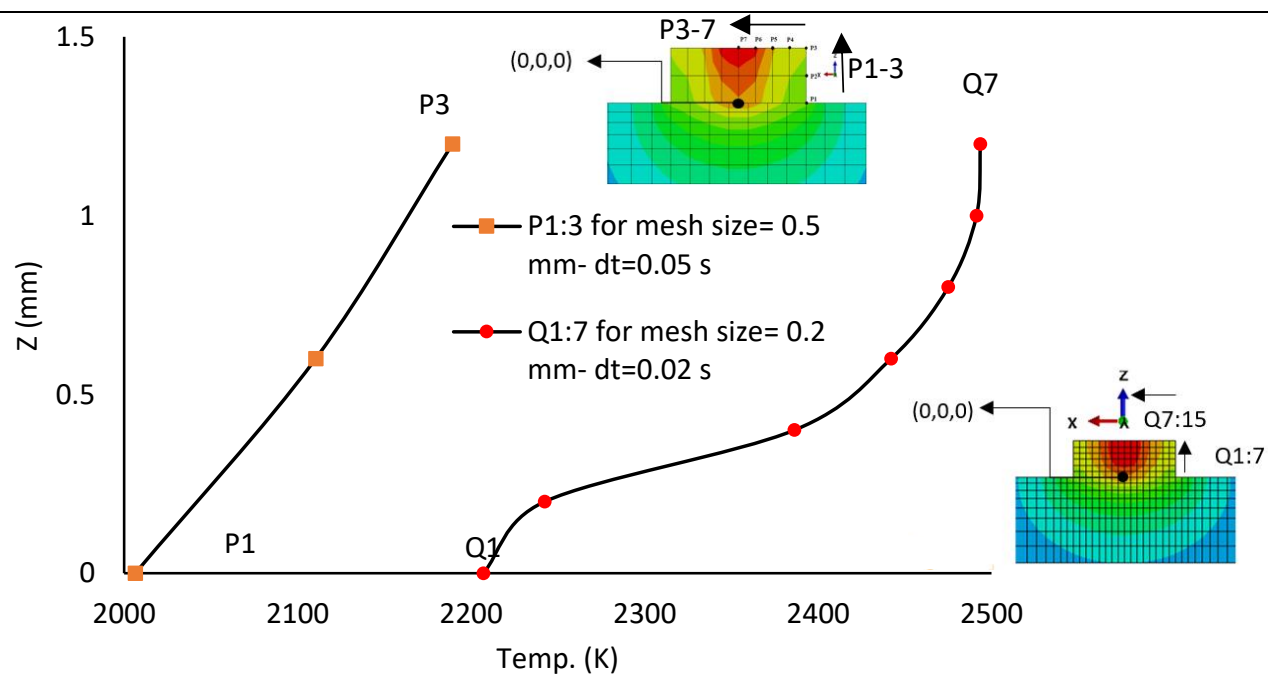




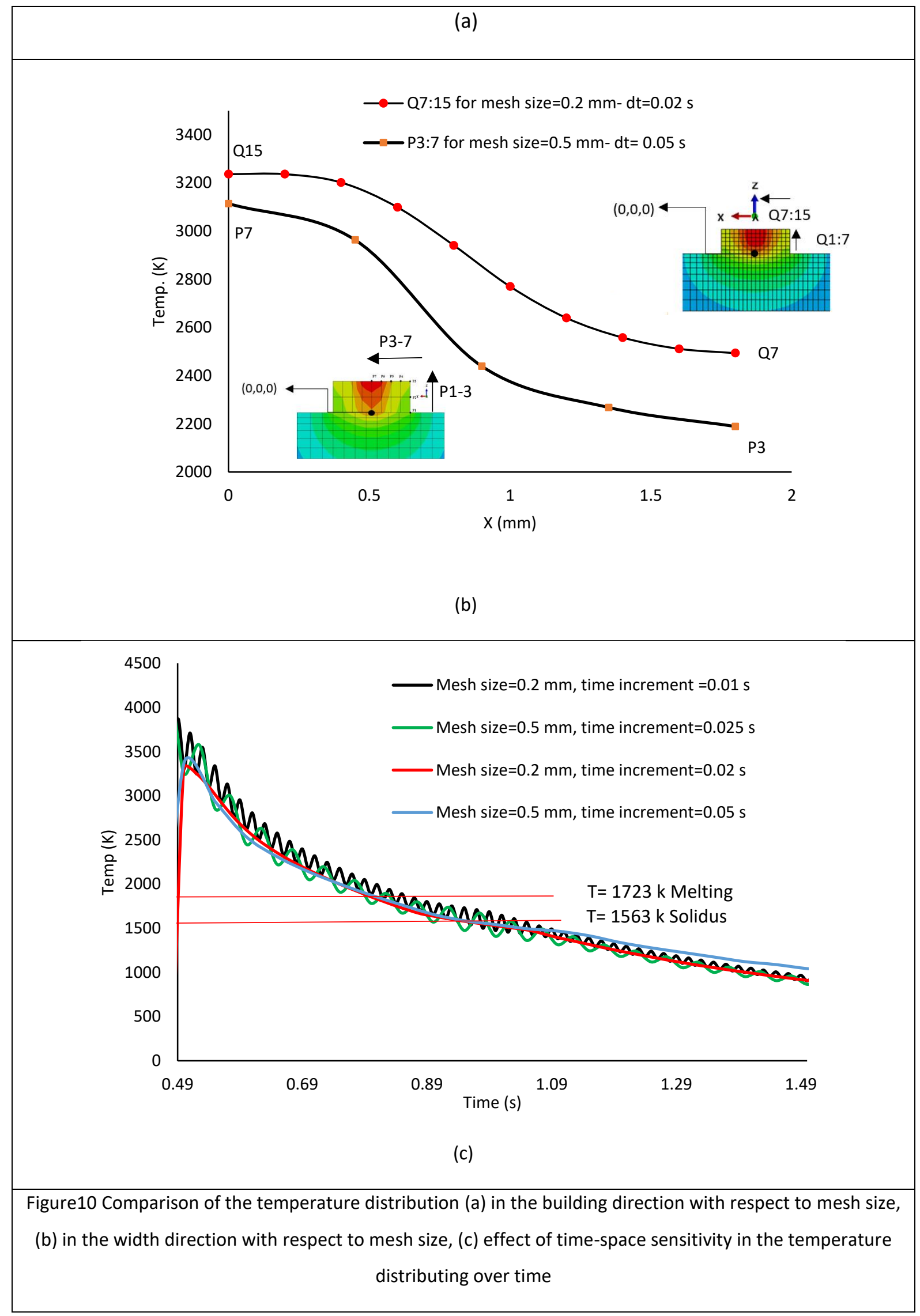




\subsection{Numerical model validation}

Three single-track laser scans are simulated firstly to validate the proposed numerical model using the experimentally measured melt pool dimensions. The processing parameters used in the simulation of is shown in Table 3. The real scanning speed, feed rate, laser power are used in the numerical model to better mimic the real phenomena. The predicted melt pool dimension using the described numerical model and the experimental results are shown in Figures 11 and 12. The results show that both melt pool dimensions including width and depth increase as more laser power is used. In other words, by increasing the laser power, the heat per unit time increases as well, and so the melt pool volume increases. The predicted results are also in good agreement with the experimental datadriven. In particular, Table 4 illustrates the comparison between width, depth of melt pool area and height of cladding lines derived from associated regression-based information experimentally and numerically.

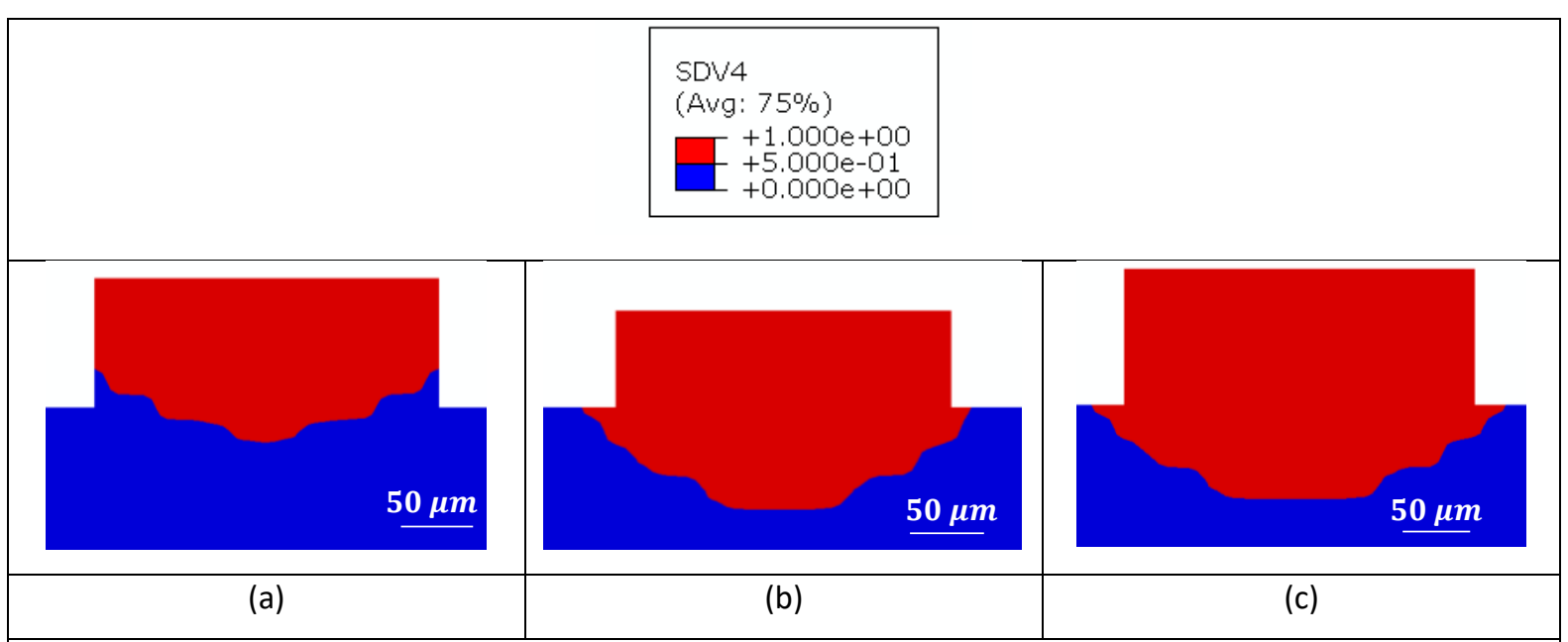

Figure11 predicted melt pool morphology in Z direction for single-track with $S S=10 \mathrm{~mm} / \mathrm{s}, \mathrm{FR}=15 \mathrm{gr} / \mathrm{min}$ (a) $P=1.5 \mathrm{~kW}$, (b) $P=2 \mathrm{~kW}$, (c) $P=2.5 \mathrm{~kW}$ 


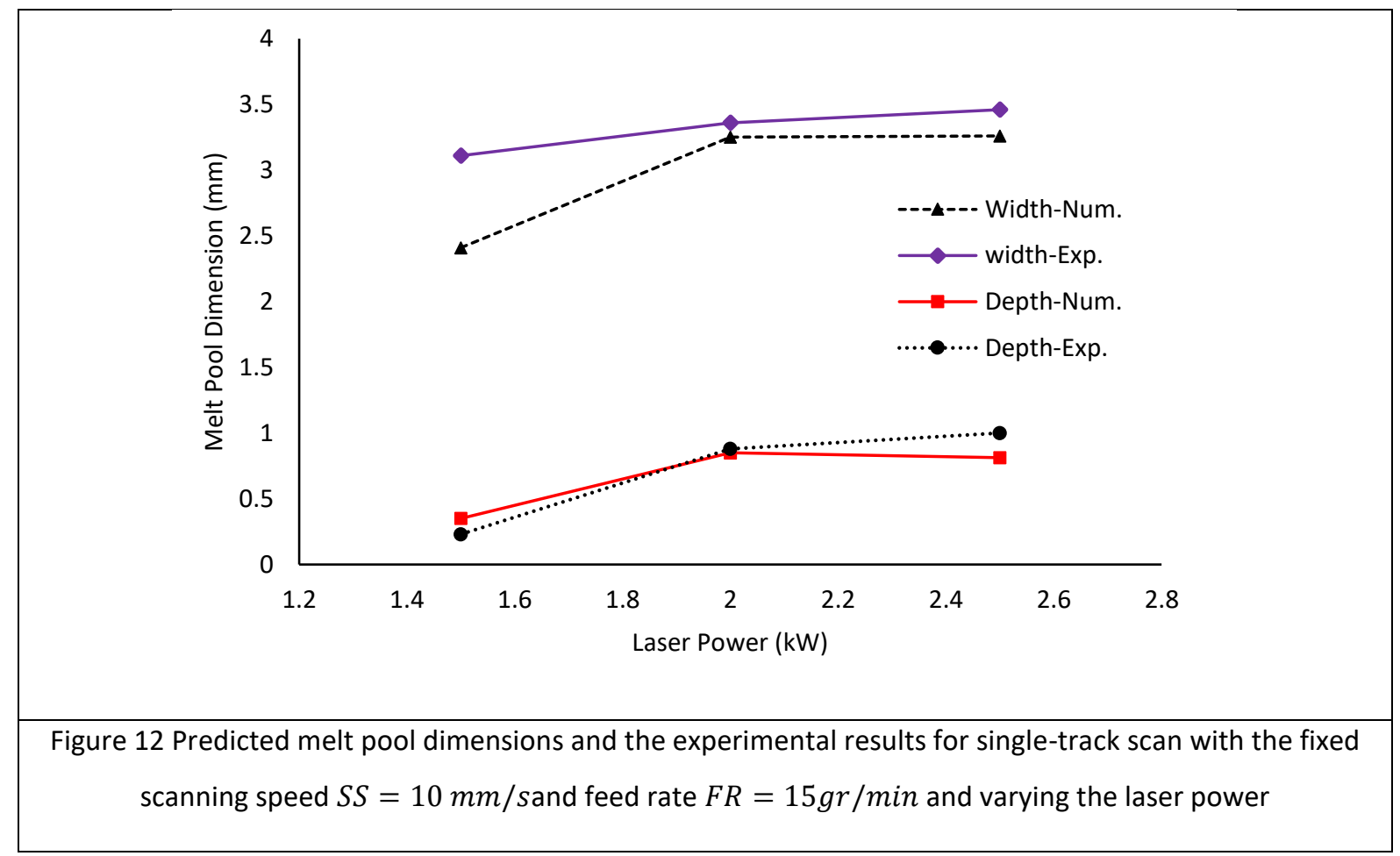

Table 4 Comparison of simulation results with experimental results

\begin{tabular}{|ccccccc|}
\hline Process Parameters & Width (Exp.) & Width (Num.) & Depth (Exp.) & Depth (Num.) & Height (Exp.) & Height (Num.) \\
\hline$P=1.5 \mathrm{~kW}, S S=10 \frac{\mathrm{mm}}{\mathrm{s}}, F R=15 \mathrm{gr} / \mathrm{min}$ & $3.11 \mathrm{~mm}$ & $2.41 \mathrm{~mm}$ & $0.23 \mathrm{~mm}$ & $0.35 \mathrm{~mm}$ & $1.03 \mathrm{~mm}$ & 1.10 \\
\hline$P=2.0 \mathrm{~kW}, S S=10 \frac{\mathrm{mm}}{\mathrm{s}}, F R=15 \mathrm{gr} / \mathrm{min}$ & $3.36 \mathrm{~mm}$ & $3.25 \mathrm{~mm}$ & $0.88 \mathrm{~mm}$ & $0.85 \mathrm{~mm}$ & $0.81 \mathrm{~mm}$ & 0.8 \\
\hline$P=2.5 \mathrm{~kW}, S S=10 \frac{\mathrm{mm}}{\mathrm{s}}, F R=15 \mathrm{gr} / \mathrm{min}$ & $3.46 \mathrm{~mm}$ & $3.26 \mathrm{~mm}$ & $1 \mathrm{~mm}$ & $0.814 \mathrm{~mm}$ & $1.23 \mathrm{~mm}$ & 1.14 \\
\hline
\end{tabular}

The red triangles and purple squares in Figure 13 show the average widths and depths of the simulated melt tracks at different scanning speeds. In the Figure, results from the simulation illustrate that both the width and depth of the melt track decrease with increasing scanning speed. Figure 14 provides evidence that by increasing the laser velocity the volume of the melt pool decrease. When the scanning speed is low, the laser remains longer around a local point, thereby generating more heat and resulting in a larger melt volume. 

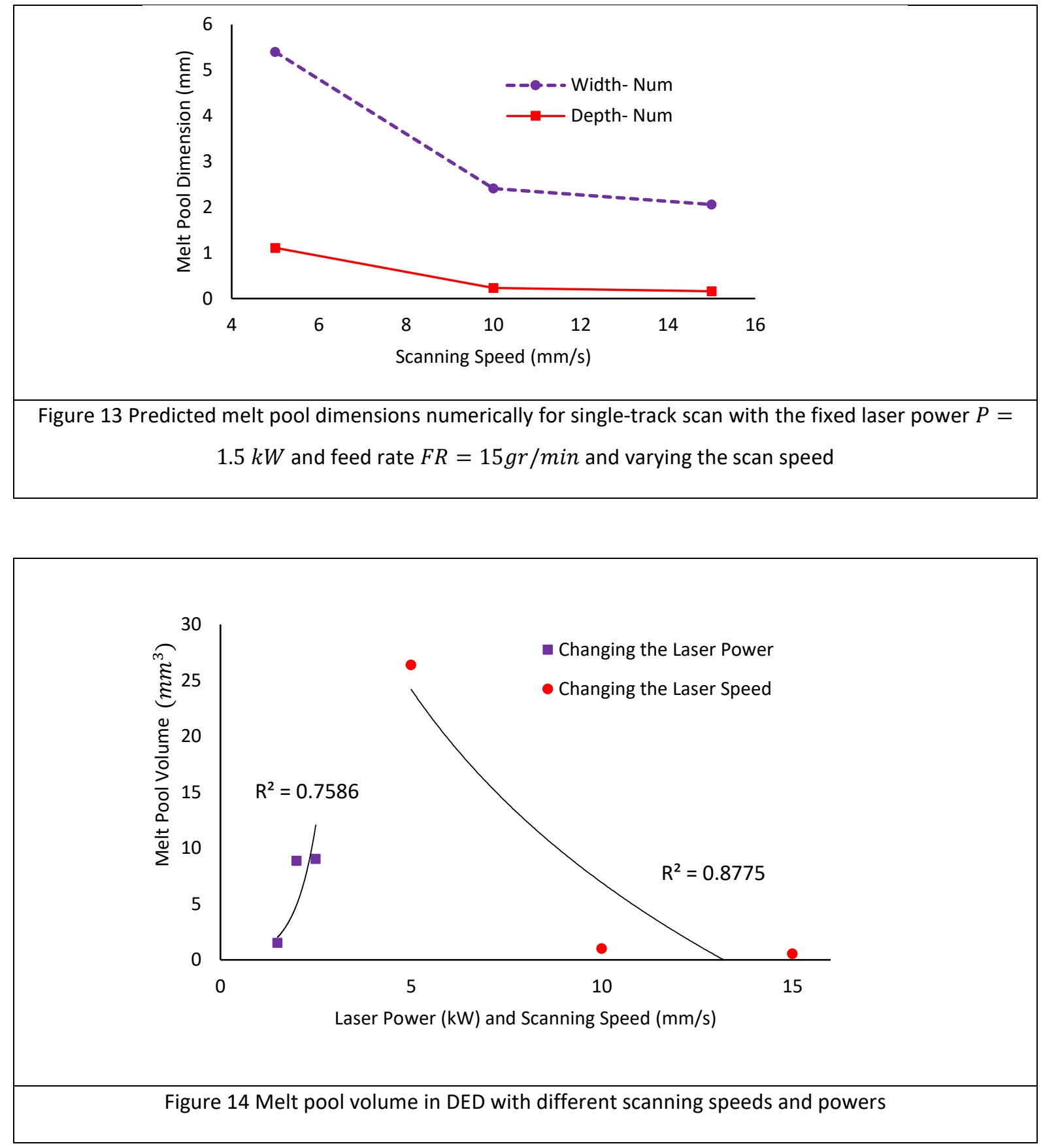

\subsection{Transient heat model associated with phase field approach}

The presented graphs in Figure $15(a-c)$ show the obtained results from the proposed model with a phase-field approach for various laser power from $1.5 \mathrm{~kW}$ to $2.5 \mathrm{~kW}$. The results indicate that by increasing the laser power the temperature value remarkably increases and even material vaporization occurs. For two laser powers $2 \mathrm{~kW}$ and $2.5 \mathrm{~kW}$ the temperature reaches vaporization.

The material phase variables include $: \phi_{f}$, with red dotted lines, which represents the time of material changes from liquid to the solid phase, the parameter $\phi_{v}$ with purple dotted line which presents a time period of vaporization phase existence, and lastly, dedicated parameter $\psi$ (black solid line) for tracking 
consolidation, between powder and melt states. Before laser reaches and starts melting $\psi$ and $\phi_{f}=$ 0 , after melting the temperature rises and material state variable $\phi_{f}$ changes to 1 which means the material state is liquid.

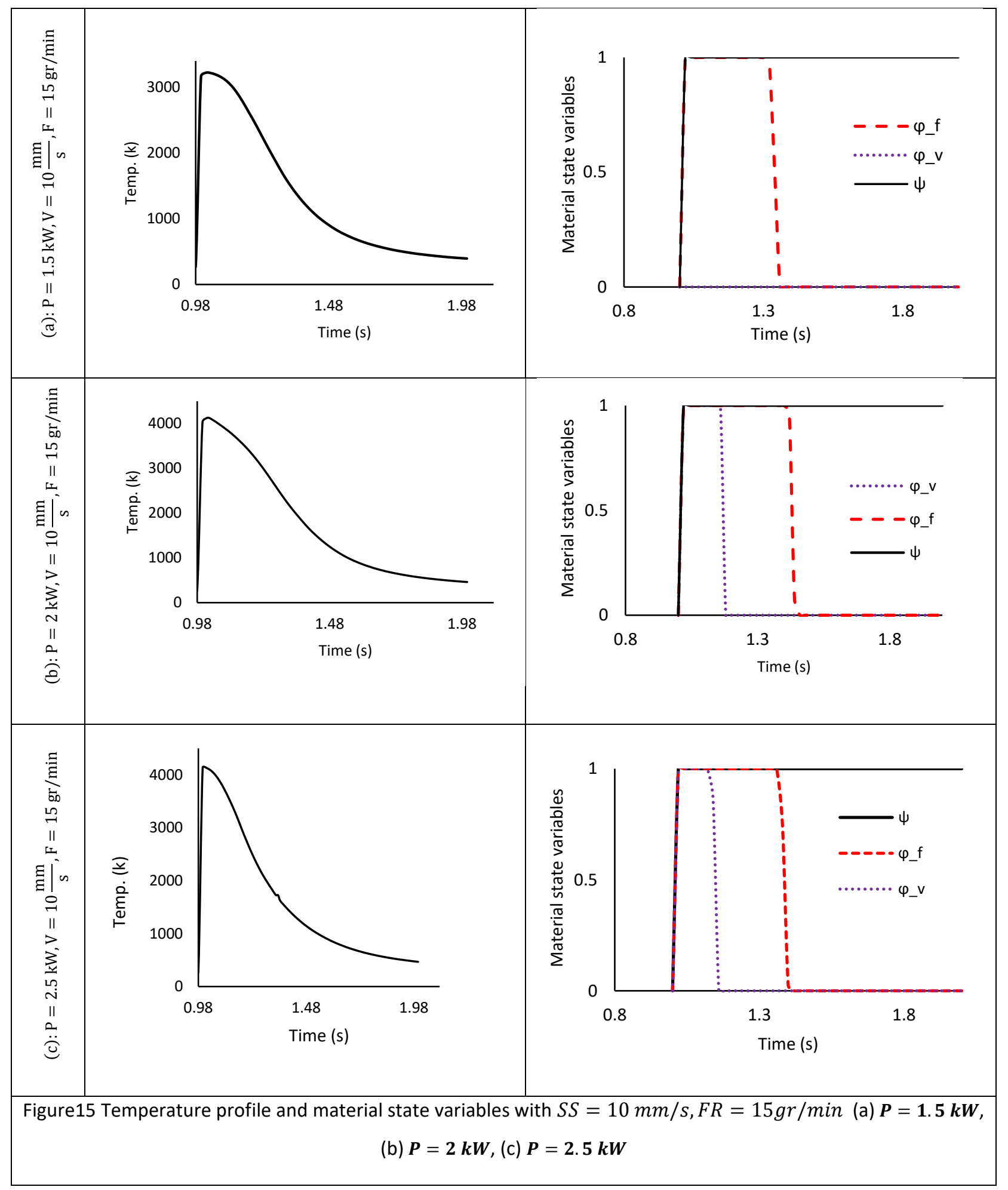

Melt pool lifetime refers to the period of time the liquid phase is present. The melt pool lifetime can be observed in Figure 16 for three different laser power values, which is an important parameter that 
determines the stability of the track formation during the process [29]. This graph illustrates when the scanning speed and feed rate are fixed and the laser power increases, the melt pool life time increases, the reason is liquid phase lifetime is not only depended on the scanning speed but also to the heat supply and volume of the melt pool. The liquid phase lifetime for laser power $1.5 \mathrm{~kW}$ is $54 \mathrm{~ms}$ and increases to $64 \mathrm{~ms}$ with laser power $2.5 \mathrm{~kW}$.

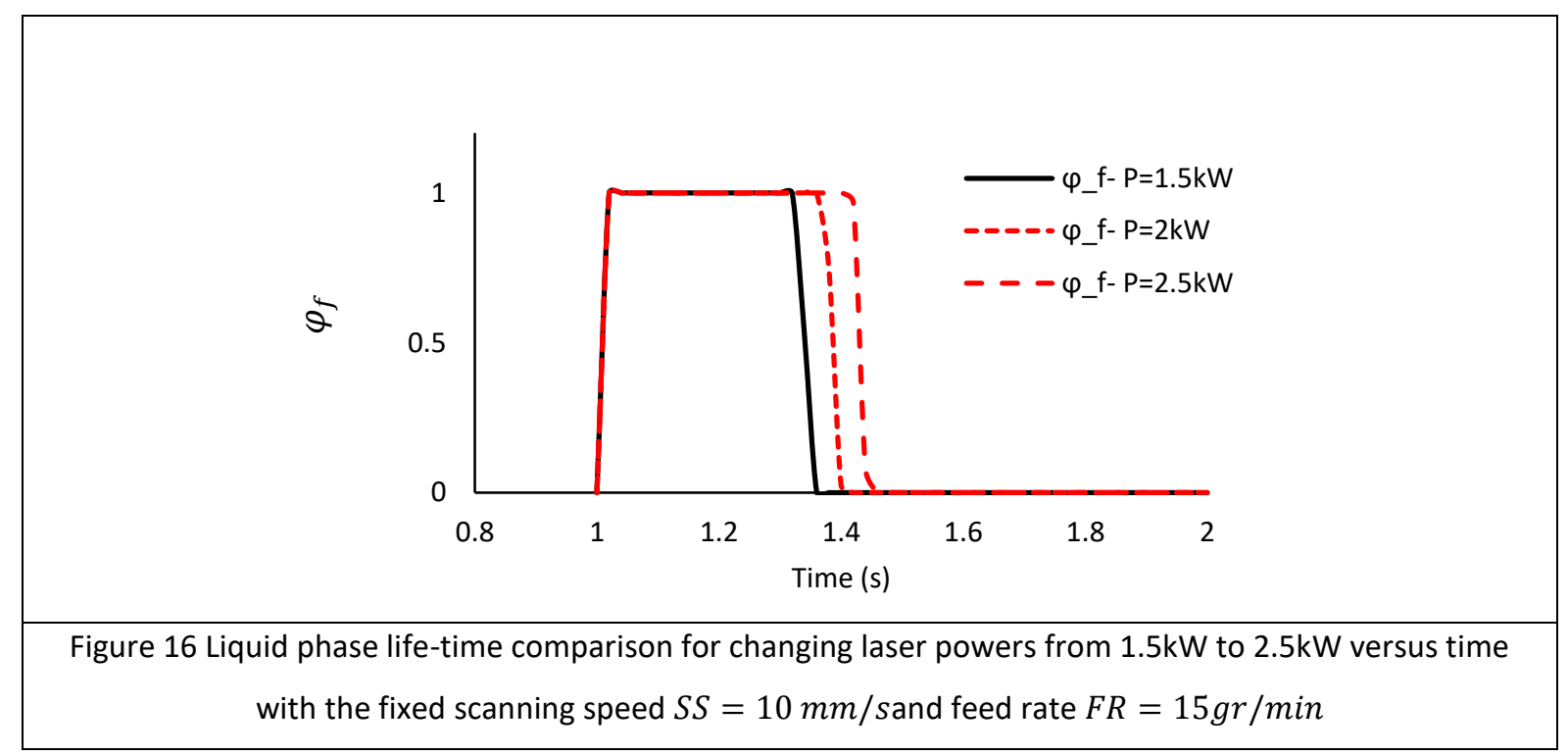

From Figure 17, the different lifetime of melt pool by varying the scanning speed while the laser power and feed rate keep fixed can be obtained. The results indicate that the melt pool lifetime tends to decrease gradually as the speed increase from $30 \mathrm{~ms}$ to $92 \mathrm{~ms}$ for $15 \mathrm{~mm} / \mathrm{s}$ and $5 \mathrm{~mm} / \mathrm{s}$ respectively.

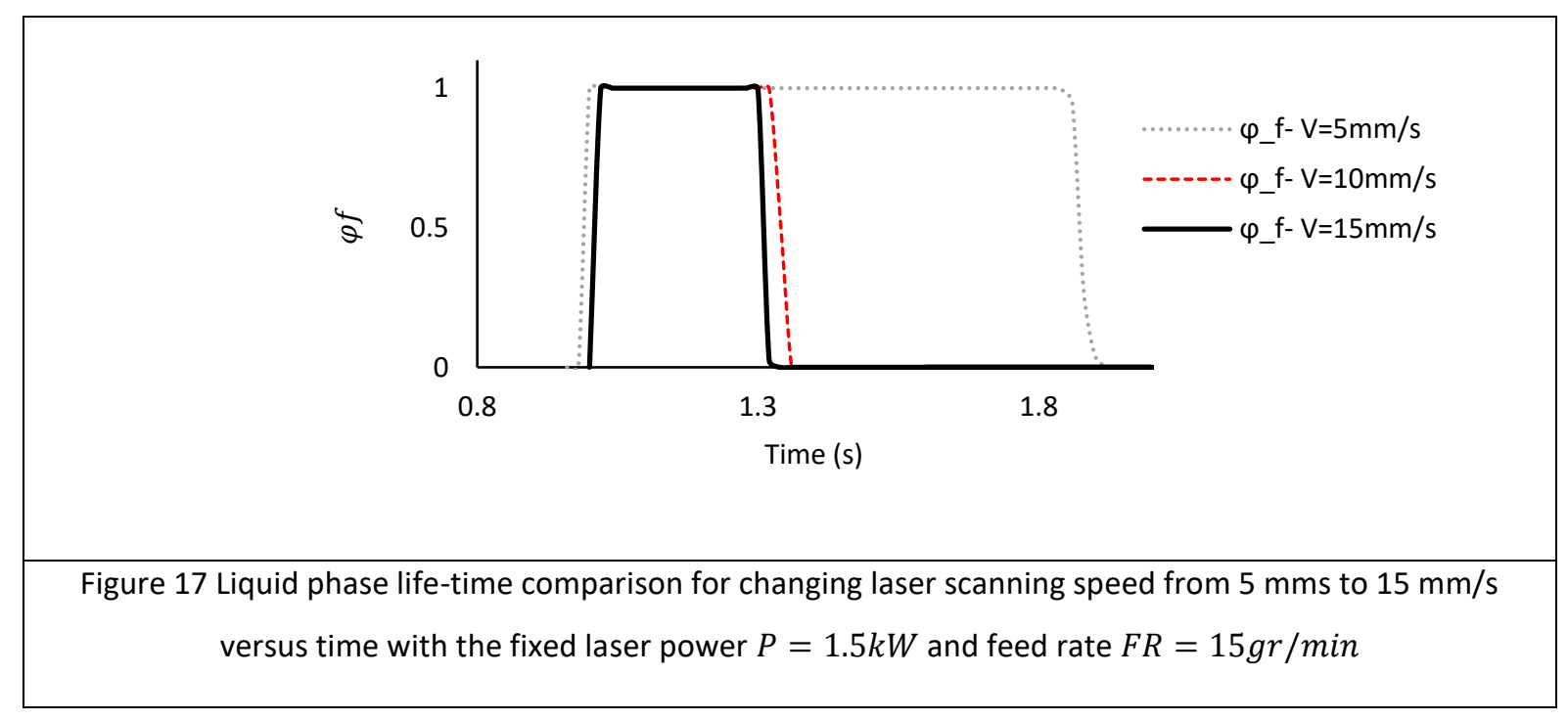

\subsection{Local temperature gradient and solidification rate $(G, R)$}

The temperature gradient $G$ and solidification growth $R$ are the most significant parameters in determining the solidification microstructure (e.g. planar, cellular, columnar dendritic, and equiaxed 
dendritic grain structure). A solidification micro graphical map for In 625 on 42CrMo4 demonstrating the variation in the morphology and size is presented in Figure 18. As can be seen the dendrite morphology, orientations, and micro segregation are different at different locations within the melt pool. This is primarily due to different positions and orientations of the initial nuclei combined with different thermal gradients and solidification velocities along the melt pool boundary.

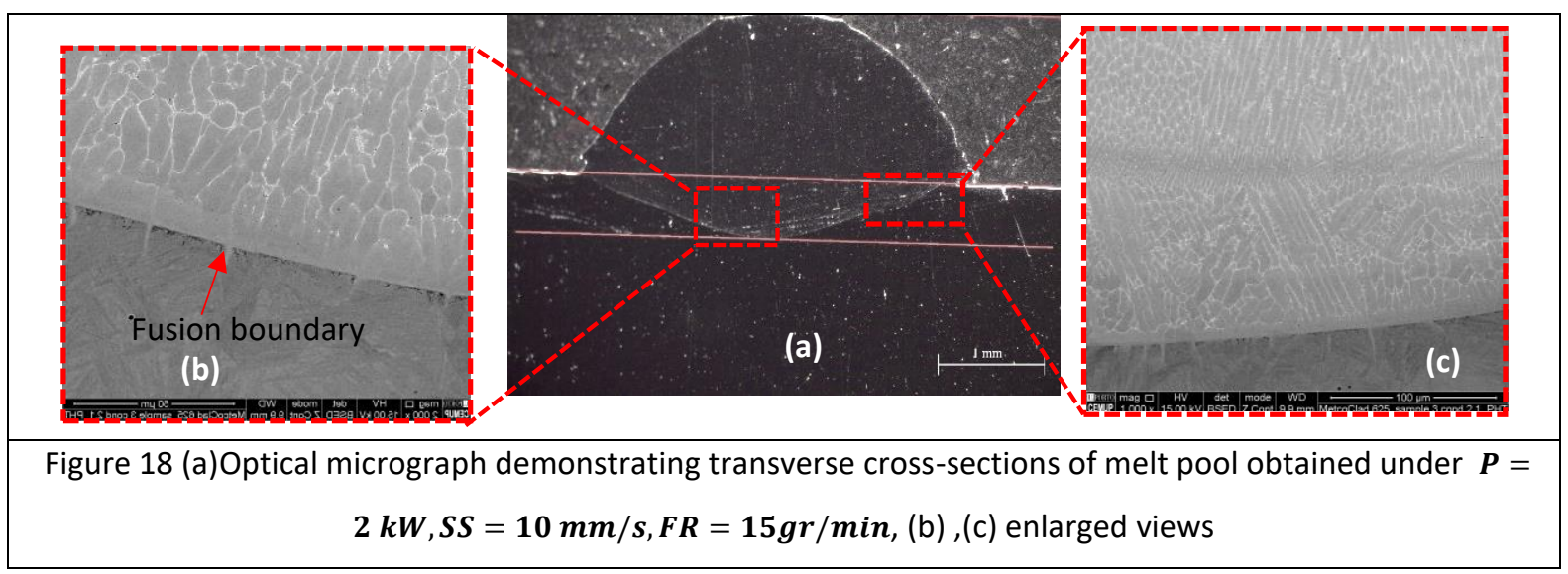

In the presented model, $G$ can be calculated from partial derivative of temperature with respect to each Cartesian coordinate as in Equation 22:

$$
G=\sqrt{\left(\frac{\partial T}{\partial x}\right)^{2}+\left(\frac{\partial T}{\partial y}\right)^{2}+\left(\frac{\partial T}{\partial z}\right)^{2}}
$$

The growth rate is geometrically derived as the projection of laser velocity $v$ onto the normal vector of solidification front, using the angle $\theta$, which is the local angle between the surface normal to the liquidus isotherm boundary and the welding direction as in Equation 23 and Figure 19;

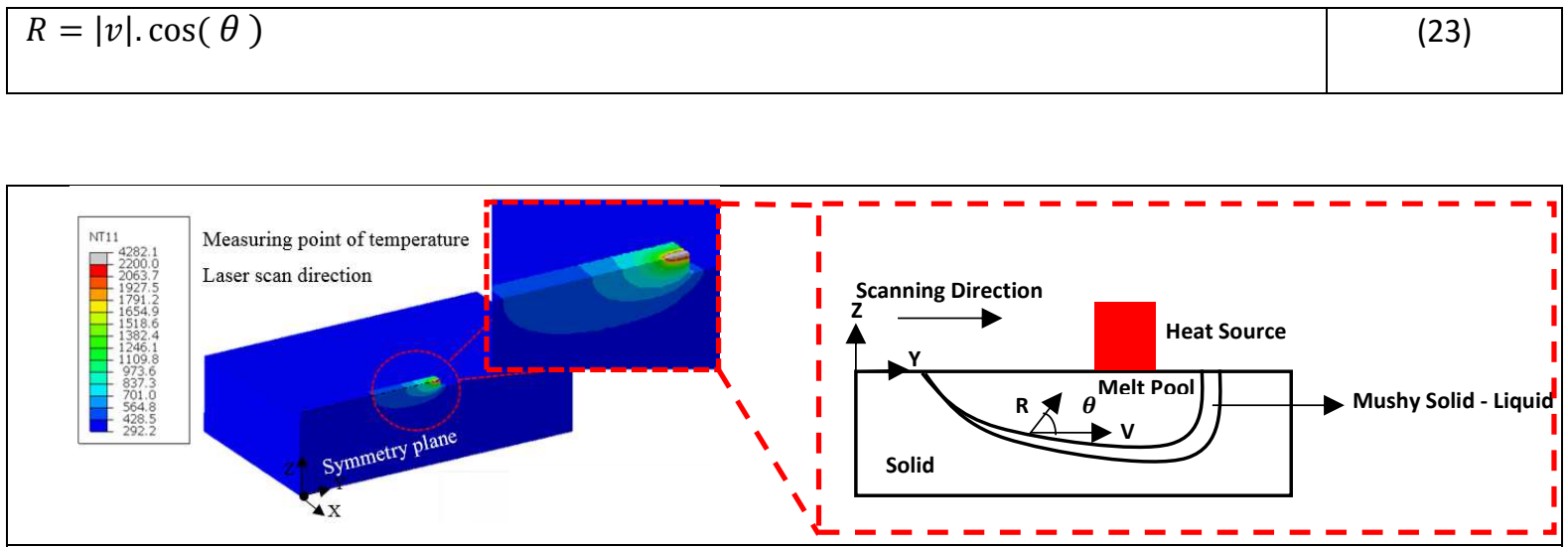

Figure 19 Schematic of solidification growth rate $R$ in the melt pool with laser scanning $V$ 
The values of $G$ and $R$ were extracted from macro-scale simulation. The points of interested were selected on the trailing half of the melt pool which is the portion subject to solidification as the melt pool domain. In Figure $20(\mathrm{a}, \mathrm{b})$, the variation of the $G$ and $R$ along with the centerline of melt pool boundary with increasing depth is explicitly shown. The results reveal that there is an inverse relationship between $R$ and $G$. The maximum $R$ is calculated near the top of the melt pool (with a low solidification front depth) while the minimum is found near the bottom of the melt pool (with a high solidification front depth). In contrast, the maximum $G$ is calculated at the bottom of the melt pool as the minimum $G$ is observed at the top. In the bottom region is exposed at high $G$ and low $R$ and hence corresponds to the planar grains as shown in Figure 18 (b) SEM microstructure evidence. On the other hand, the top regions tend relatively lower $G$ and higher $R$ with the dendrite structure from Figure 18 (c).

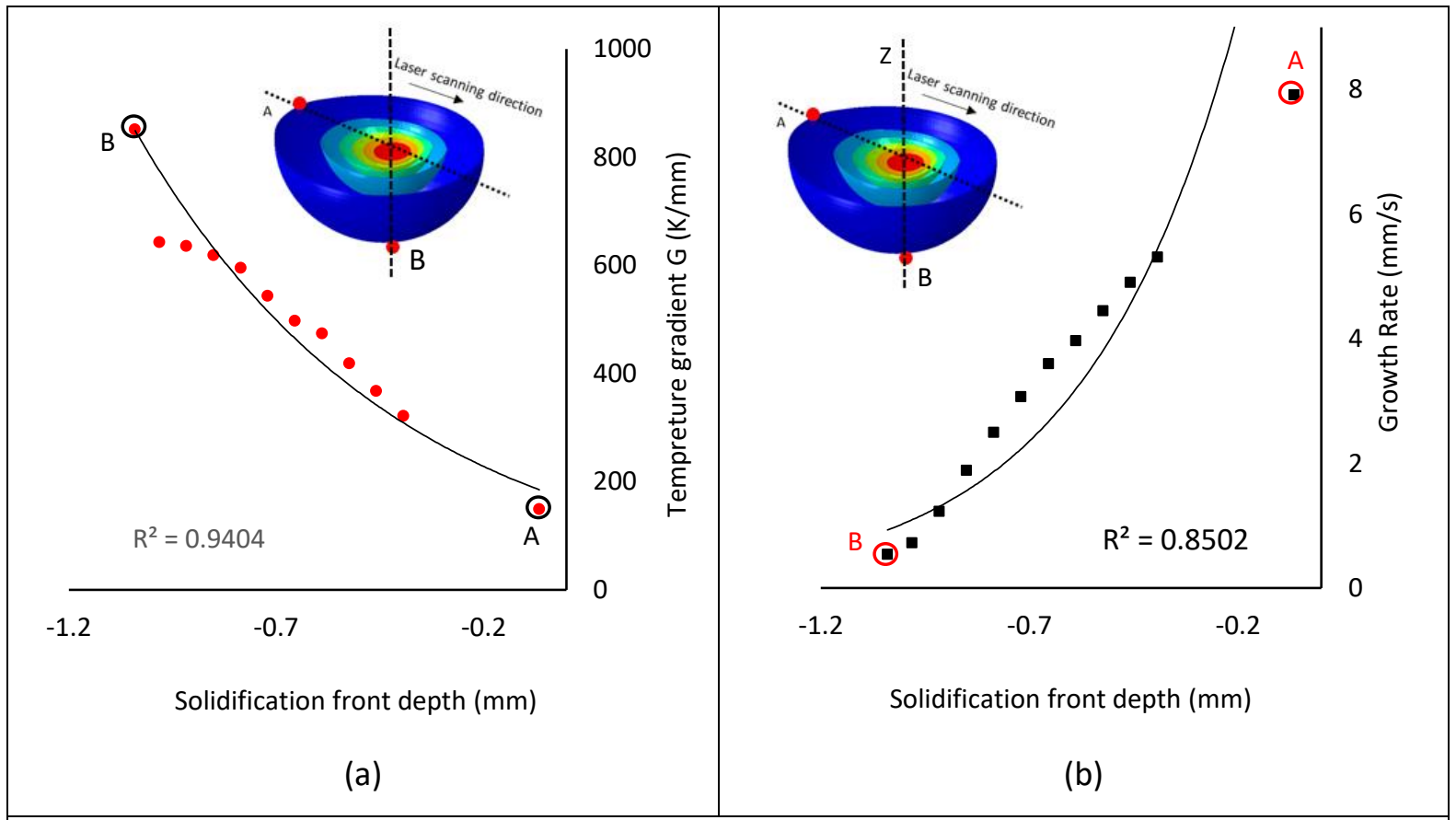

Figure 20 The calculated (a): $G$ and (b): $R$, along the centerline of the melt pool surface; Variation in relation with the solidification front depth for one set of parameters $P=2 \mathrm{~kW}, S S=10 \mathrm{~mm} / \mathrm{s}, F R=15 \mathrm{gr} / \mathrm{min}$

Figure $21(\mathrm{a}, \mathrm{b})$ illustrates the cooling rate for two set of parameters including (a): $\boldsymbol{P}=\mathbf{2} \boldsymbol{k W}, \boldsymbol{S S}=$ $10 \mathrm{~mm} / \mathrm{s}, F R=15 \mathrm{gr} / \mathrm{min}$ and (b): $P=1.5 \mathrm{~kW}, S S=10 \mathrm{~mm} / \mathrm{s}, F R=15 \mathrm{gr} / \mathrm{min}$. It can be seen how the cooling rate, $\dot{T}=G \times R$, varies as a function of the melt pool depth for both cases; the dotted rectangular views emphasize the highest and lowest cooling rate along with the location of the melt pool boundary. Simulation results show that cooling rate increases from bottom to top along the melt pool boundary. The predicted cooling rate varies by changing the laser power. The cooling rate 
increases with decreasing the laser power [20][30] and consequently differences in cooling rate leads to variety of solidified microstructures.

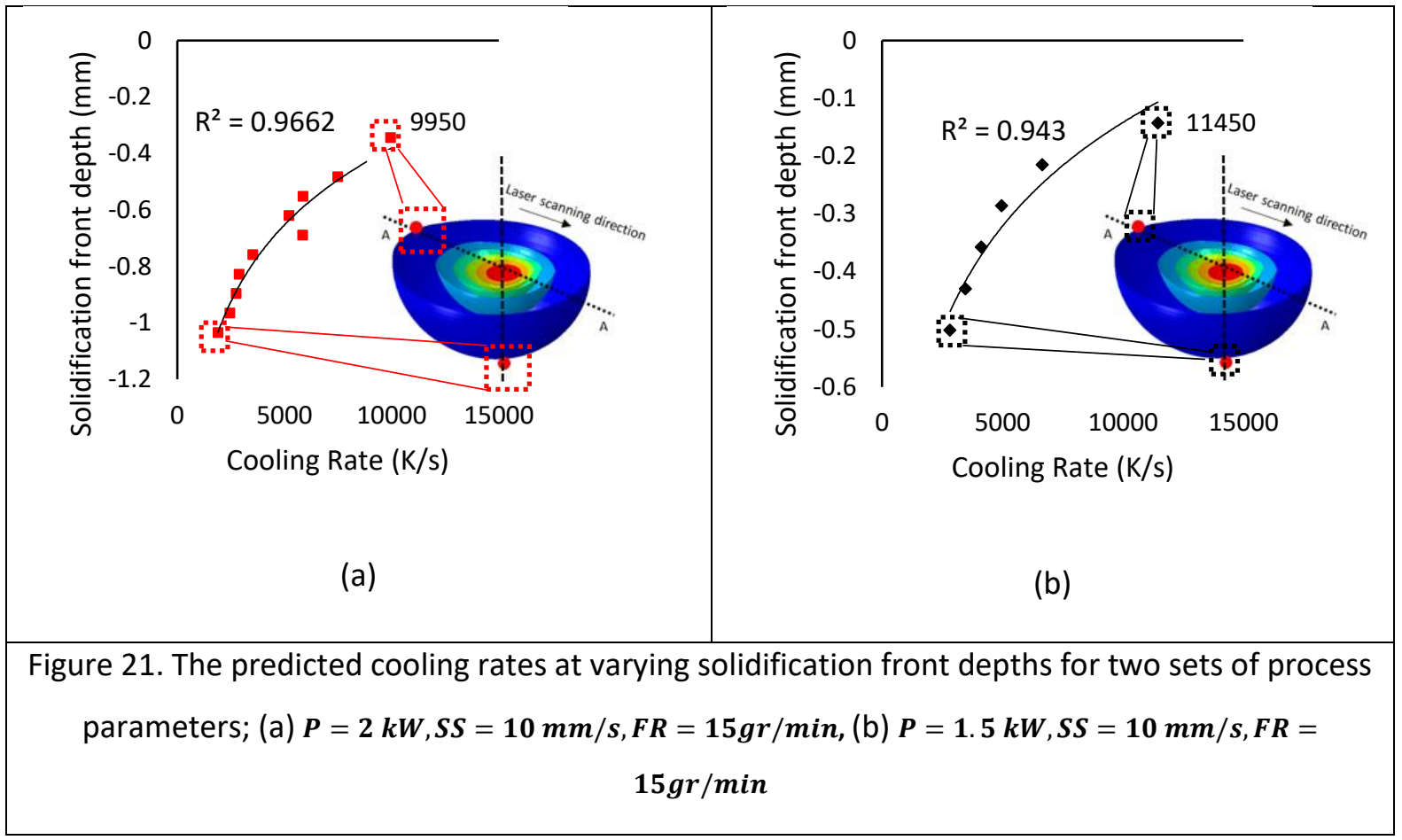

\section{Conclusion}

In the present study, a computational framework for the transient heat phenomena in DED process is developed by coupling a finite element based on phase-field model. In the proposed model three state variables $\phi_{f}, \phi_{v}$, and $\psi$ are defined to track and capture the phase of materials between molten, solid, and even vaporized conditions to allocate the proper thermal-physical material properties. The proposed model is implemented in the commercial finite element software ABAQUS-standard. The capability of numerical model is investigated by comparing melt pool dimensions, including width and depth from simulation for single cladding lines, with the experimental data. Three different laser power values were applied to quantify their effect on the melt pool morphology. The melt pool width and depth have a tendency of becoming greater when a higher laser power is applied. Moreover, the numerical results showcases the inverse correlation between the scanning speed the melt pool volume. The investigation is further proceeded to detect the solidification parameters, the temperature gradient and the growth rate. To achieve this goal, the temperature variation as well as the liquidus isotherm boundary surface information is utilized to calculate these parameters along the solidification front depth path. The planar to dendrite transition was predicted by moving from melt pool boundary to the interior of melt pool. Furthermore, an inverse relationship between $R$ and $G$, can be seen and maximum $R$ is calculated near the top of the melt pool, while the minimum is found near the bottom of the melt pool, which is in agreement with a solidification map obtained by SEM. 
Finally, the cooling rate increases from the bottom to the top of melt pool and decreasing laser power leads to an increase of the range of the cooling rate.

Availability of data and materials: It has been confirmed that data is open and transparent

Code availability: Not applicable

Funding: This work was supported by Fundo Europeu de Desenvolvimento Regional (FEDER) through Programa Operacional Competitividade e Internacionalização (COMPETE 2020) and national funds through Fundação para a Ciência e a Tecnologia (FCT), Portugal. Grant number : ADDing (POCI-010145-FEDER-030490)

\section{Declarations:}

\section{Author contribution:}

Roya Darabi: Conceptualization, investigation, writing—original draft, writing—review and editing Andre Ferreira: Experimental coordinator

Erfan Azinpour: Writing-review and editing

Jose Cesar de Sa: Conceptualization, investigation, review and editing, funding acquisition

Ana Reis: Conceptualization, investigation, review and editing, funding acquisition

Ethics approval Not applicable

Consent to participate Not applicable

Consent for publication Not applicable

Declaration of conflicting interests:

The author(s) declared no potential conflicts of interest with respect to the research, authorship, and/or publication of this article.

Competing interests The authors declare no competing interests. 


\section{References}

1. DebRoy, T.; Wei, H.L.; Zuback, J.S.; Mukherjee, T.; Elmer, J.W.; Milewski, J.O.; Beese, A.M.; Wilson-Heid, A.; De, A.; Zhang, W. Additive manufacturing of metallic components - Process, structure and properties. Prog. Mater. Sci. 2018, 92, 112-224, doi:10.1016/j.pmatsci.2017.10.001.

2. Azinpour, E.; Darabi, R.; Cesar de Sa, J.; Santos, A.; Hodek, J.; Dzugan, J. Fracture analysis in directed energy deposition (DED) manufactured 316L stainless steel using a phase-field approach. Finite Elem. Anal. Des. 2020, 177, 103417, doi:10.1016/j.finel.2020.103417.

3. Gardan, J. Additive manufacturing technologies: State of the art and trends. Int. J. Prod. Res. 2016, 54, 3118-3132, doi:10.1080/00207543.2015.1115909.

4. Gibson, D.G.; Glass, J.I.; Lartigue, C.; Noskov, V.N.; Chuang, R.Y.; Algire, M.A.; Benders, G.A.; Montague, M.G.; Ma, L.; Moodie, M.M.; et al. Creation of a bacterial cell controlled by a chemically synthesized genome. Science (80-. ). 2010, 329, 52-56, doi:10.1126/science.1190719.

5. Somashekara, M.A.; Naveenkumar, M.; Kumar, A.; Viswanath, C.; Simhambhatla, S. Investigations into effect of weld-deposition pattern on residual stress evolution for metallic additive manufacturing. Int. J. Adv. Manuf. Technol. 2017, 90, 2009-2025, doi:10.1007/s00170-016-9510-7.

6. Gu, D.D.; Meiners, W.; Wissenbach, K.; Poprawe, R. Laser additive manufacturing of metallic components: Materials, processes and mechanisms. Int. Mater. Rev. 2012, 57, 133-164, doi:10.1179/1743280411Y.0000000014.

7. Feenstra, D.R.; Banerjee, R.; Fraser, H.L.; Huang, A.; Molotnikov, A.; Birbilis, N. Critical review of the state of the art in multi-material fabrication via directed energy deposition. Curr. Opin. Solid State Mater. Sci. 2021, 25, 100924, doi:10.1016/j.cossms.2021.100924.

8. Fallah, V.; Alimardani, M.; Corbin, S.F.; Khajepour, A. Temporal development of melt-pool morphology and clad geometry in laser powder deposition. Comput. Mater. Sci. 2011, 50, 2124-2134, doi:10.1016/j.commatsci.2011.02.018.

9. Lee, K.H.; Yun, G.J. A novel heat source model for analysis of melt Pool evolution in selective laser melting process. Addit. Manuf. 2020, 36, 101497, doi:10.1016/j.addma.2020.101497.

10. Lee, K.H.; Yun, G.J. Prediction of melt pool dimension and residual stress evolution with thermodynamicallyconsistent phase field and consolidation models during re-melting process of SLM. Comput. Mater. Contin. 2021, 66, 87-112, doi:10.32604/cmc.2020.012688.

11. Roy, S.; Juha, M.; Shephard, M.S.; Maniatty, A.M. Heat transfer model and finite element formulation for simulation of selective laser melting. Comput. Mech. 2018, 62, 273-284, doi:10.1007/s00466-017-1496-y.

12. Markl, M.; Körner, C. Multiscale Modeling of Powder Bed-Based Additive Manufacturing. Annu. Rev. Mater. Res. 2016, 46, 93-123, doi:10.1146/annurev-matsci-070115-032158.

13. Proell, S.D.; Wall, W.A.; Meier, C. On phase change and latent heat models in metal additive manufacturing process simulation; Springer International Publishing, 2020; Vol. 7; ISBN 0123456789.

14. Darabi, R.; Azinpour, E. Fracture Prediction Based on Evaluation of Initial Porosity Induced By Direct Energy Deposition. 2021, doi:10.13052/ejcm2642-2085.29233. 
15. Wolff, S.J.; Lin, S.; Faierson, E.J.; Liu, W.K.; Wagner, G.J.; Cao, J. A framework to link localized cooling and properties of directed energy deposition (DED)-processed Ti-6Al-4V. Acta Mater. 2017, 132, 106-117, doi:10.1016/j.actamat.2017.04.027.

16. Farshidianfar, M.H.; Khajepour, A.; Gerlich, A.P. Effect of real-time cooling rate on microstructure in Laser Additive Manufacturing. J. Mater. Process. Technol. 2016, 231, 468-478, doi:10.1016/j.jmatprotec.2016.01.017.

17. Wang, Z.; Palmer, T.A.; Beese, A.M. Effect of processing parameters on microstructure and tensile properties of austenitic stainless steel 304L made by directed energy deposition additive manufacturing. Acta Mater. 2016, 110, 226-235, doi:10.1016/j.actamat.2016.03.019.

18. Hagenlocher, C.; Fetzer, F.; Weller, D.; Weber, R.; Graf, T. Explicit analytical expressions for the influence of welding parameters on the grain structure of laser beam welds in aluminium alloys. Mater. Des. 2019, 174, 107791, doi:10.1016/j.matdes.2019.107791.

19. Karayagiz, K.; Johnson, L.; Seede, R.; Attari, V.; Zhang, B.; Huang, X.; Ghosh, S.; Duong, T.; Karaman, I.; Elwany, A.; et al. Finite interface dissipation phase field modeling of $\mathrm{Ni}-\mathrm{Nb}$ under additive manufacturing conditions. Acta Mater. 2020, 185, 320-339, doi:10.1016/j.actamat.2019.11.057.

20. Sekerka, R.F.; Wheeler, A.A. for Solidification. 1992.

21. Reis, A.; Xu, Z.; Tol, R. V.; Neto, R. Modelling feeding flow related shrinkage defects in aluminum castings. J. Manuf. Process. 2012, 14, 1-7, doi:10.1016/j.jmapro.2011.05.003.

22. Reis, A.; Houbaert, Y.; Xu, Z.; Van Tol, R.; Santos, A.D.; Duarte, J.F.; Magalhães, A.B. Modeling of shrinkage defects during solidification of long and short freezing materials. J. Mater. Process. Technol. 2008, 202, 428-434, doi:10.1016/j.jmatprotec.2007.10.030.

23. Guo, Z.; Lin, P. A thermodynamically consistent phase-field model for two-phase flows with thermocapillary effects. J. Fluid Mech. 2015, 766, 226-271, doi:10.1017/jfm.2014.696.

24. Zhang, Z.; Huang, Y.; Rani Kasinathan, A.; Imani Shahabad, S.; Ali, U.; Mahmoodkhani, Y.; Toyserkani, E. 3Dimensional heat transfer modeling for laser powder-bed fusion additive manufacturing with volumetric heat sources based on varied thermal conductivity and absorptivity. Opt. Laser Technol. 2019, 109, 297-312, doi:10.1016/j.optlastec.2018.08.012.

25. Roberts, I.A.; Wang, C.J.; Esterlein, R.; Stanford, M.; Mynors, D.J. A three-dimensional finite element analysis of the temperature field during laser melting of metal powders in additive layer manufacturing. Int. J. Mach. Tools Manuf. 2009, 49, 916-923, doi:10.1016/j.ijmachtools.2009.07.004.

26. Katopodes, N.D.; Method, E.E.; Size, S.; Condition, B.; Factor, A.; Cell, D. low-Water Flow Methods for TwoDimensional Shal- Courant Number.

27. Deering, R.A. Additive Manufacturing Part Level Distortion Sensitivity Analysis within Abaqus on a Thin Walled, Tubular Structure. Addit. Manuf. Symp. 2018, 14.

28. Yashchuk, I. A multiscale finite element framework for additive manufacturing process modeling. 2018.

29. Haley, J.C.; Zheng, B.; Bertoli, U.S.; Dupuy, A.D.; Schoenung, J.M.; Lavernia, E.J. Working distance passive stability in laser directed energy deposition additive manufacturing. Mater. Des. 2019, 161, 86-94, doi:10.1016/j.matdes.2018.11.021. 
30. Ali, H.; Ghadbeigi, H.; Mumtaz, K. Processing Parameter Effects on Residual Stress and Mechanical Properties of Selective Laser Melted Ti6Al4V. J. Mater. Eng. Perform. 2018, 27, 4059-4068, doi:10.1007/s11665-018-3477-5. 